\title{
Existence of Steady State Solutions with Finite Energy for the Magnetohydrodynamic Equations in the Whole Space $\mathbf{R}^{3}$
}

\author{
P.D.Raiter ${ }^{1}$ and R.V.Saraykar ${ }^{2}$ \\ ${ }^{1}$ Department of Mathematics ,RTM Nagpur University, University Campus ,Nagpur-440033,India. \\ ${ }^{2}$ Department of Mathematics ,RTM Nagpur University, University Campus ,Nagpur-440033,India.
}

\begin{abstract}
We study the steady state Magnetohydrodynamic (MHD) equations in the whole space $R^{3}$. Following the work of C. Bjorland and M. Schonbek [4] onNavier-Stokes equations in the whole space, we prove the existence of atleast one solution with finite Dirichlet Integral $(\|(\nabla U, \nabla B)\|<\infty)$ to steady state Magnetohydrodynamic equations in the whole space. Further, we show that these solutions are unique among all solutions with finite energy and finite DirichletIntegral .
\end{abstract}

\section{Introduction}

Magnetohydrodynamics (MHD) is the study of flows of fluids which are electrically conducting and move in a magnetic field. The simplest example of an electrically conducting fluid is a liquid metal like mercury or liquid sodium. MHD treats, in particular, conducting fluids either in liquid form or gaseous form. The equations describing the motion of a viscous incompressible conducting fluid moving in a magnetic field are derived by coupling Navier-Stokes equations with Maxwell's equations together with expression for the Lorentz force. The domain $\Omega$ in which the fluid is moving is either a bounded subset of $R^{3}$ or the whole space $R^{3}$. In this paper we restrict our considerations to a domain $\Omega$ which is the whole space $R^{3}$.During past four or five decades, there have been an extensive study of qualitativeproperties such as existence, uniqueness, regularity and stability of solutions of theMHD equations. This is evident from the work of Duvaut and Lions [1], E. SanchezPalencia [2], Sermange and Temam [3] and other researchers working in the field. Themethods from nonlinear functional analysis such as Galerkin approximation, fixed pointtheorems, monotone and coercive operators, semigroup theory etc have been applied toestablish many a qualitative properties for compressible as well as incompressible MHDflows. The function spaces used are either Holder spaces or Sobolev spaces which are theappropriate function spaces for using these methods and the theory of elliptic operators.

In spite of these works, there are very few qualitative results available in the case where the domain is the full space. In the case when domain is a bounded subset of $\mathrm{R}^{3}$, it is easy to obtain qualitative results by using Poincare type inequality. But for unbounded domain, one has to use other techniques as were developed by C. Bjorland and M. Schonbek [4]. As for MHD flows for incompressible conducting fluids, there are other works where regularity results for MHD flows have been proved ( see references [5-7] ). However, as in the case of Navier-Stokes equations for incompressible fluids, the proof of global regularity remains illusive in this case also. In the present paper, we show that the techniques used in [4] can be extended to prove similar results for steady state Magnetohydrodynamic (MHD) flows.

Thus, we consider viscous incompressible Magnetohydrodynamic (MHD) flow governed by the following equations: 


$$
\left.\begin{array}{l}
\mathrm{u}_{t}-v \Delta u+\sum_{i=1}^{n} u_{i} D_{i} u-\sum_{i=1}^{n} b_{i} D_{i} b+\operatorname{grad} p=f \text { in } \Omega \\
\mathrm{b}_{t}-\lambda \Delta b+\sum_{i=1}^{n} u_{i} D_{i} b-\sum_{i=1}^{n} b_{i} D_{i} u=0 \text { in } \Omega \\
\nabla \cdot u=\left.0 \quad(u, b)\right|_{\partial \Omega}(0,0)=\left(u_{0}, b_{0}\right) \\
\nabla \cdot b=0
\end{array}\right\}
$$

describing the motion of a conducting fluid in an electromagnetic field, where $u=u(t, x)=u\left(t, x_{1}, x_{2}, x_{3}\right)$ is the velocity vector, $\mathrm{b}=\mathrm{b}(\mathrm{t}, \mathrm{x})$ is the magnetic field vector, $v$ is the kinematic co-efficient of viscosity, $\lambda$ is the co-efficient of magnetic diffusivity,

$p=p(t, x)$ is the pressure, $f=f(t, x)$ is the external force, $\left(u_{0}, b_{0}\right)(x)=R^{3}$ is the initial condition,$\Omega$ is a domain which is a bounded subset of $R^{3}$ and $\partial \Omega$ denotes the boundary of $\Omega$. In the present paper ,we are interested in steady state solution for the MHD equations. For this we consider the following system of partial differential equations :

$$
\begin{aligned}
& \sum_{i=1}^{n} U_{i} D_{i} U-\sum_{i=1}^{n} B_{i} D_{i} U+\operatorname{grad} p=v \Delta U+f \\
& \sum_{i=1}^{n} U_{i} D_{i} B-\sum_{i=1}^{n} B_{i} D_{i} U=\lambda \Delta B \ldots(1.2 \mathrm{~B}) \\
& \nabla \cdot U=0, \nabla \cdot B=0
\end{aligned}
$$

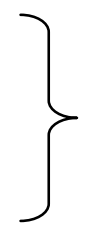

Such partial differential equations are satisfied by those solutions for which $\partial_{t} U=0$ and $\partial_{t} B=0$ which means the solution is constant with respect to time. Thus, the solution $(U, B)$ is the steady state solution of the MHD equations satisfying $\partial_{t} U=0$ and $\partial_{t} B=0$ respectively .

As we are working with functions with compact support we can say that $(U, B)$ tends to zero as $|x|$ becomes large.

We denote by $L^{2}(\Omega) \quad$ the space of square integrable functions and all integrals in this paper are taken over the whole space $R^{3}$ unless otherwise stated, $C_{0}^{\infty}$ denotes the space of smooth functions with compact support woreover have:

$$
\begin{aligned}
& \langle f, g\rangle=\int f \cdot g \quad V=\left\{\varphi \in C_{0}^{\infty} \mid \nabla \cdot \varphi=o\right\} \\
& \|\cdot\|_{p}=\left(\int|\cdot|^{p}\right)^{1 / p} \quad L_{\sigma}^{p}=\left\{\text { completion of } V \text { under the norm }\|\cdot\|_{p}\right\} \\
& \|\cdot\|_{\dot{H}_{1}}=\|\nabla \cdot\|_{2} \stackrel{\circ}{H}_{\sigma}^{1}=\left\{\text { completion of } V \text { under the norm }\|\cdot\|_{\dot{H}_{1}}\right\} \\
& H_{\sigma}^{1}=L_{\sigma}^{2} \cap \stackrel{\circ}{H}_{\sigma}^{1}\left(\stackrel{\circ}{H}_{\sigma}^{1}\right)^{\prime}=\left\{\text { dual of }{\stackrel{\circ}{H_{\sigma}}}^{1}\right\} \\
& \left.X=L_{\sigma}^{2} \cap\left(\stackrel{\circ}{H}_{\sigma}^{1}\right)^{\prime}\left\{\|\cdot\|_{x}=\max \left\{\|\cdot\|_{2},\|\cdot\|_{\left(\stackrel{\circ}{H}_{\sigma}^{\prime}\right.}\right)^{1}\right\}\right\} \\
& \hat{f}(\xi)=\int f(x) e^{-2 \pi i x \cdot \xi} d x \\
& f(x)=\int f(\xi) e^{2 \pi i x \cdot \xi} d \xi
\end{aligned}
$$

Furthermore, we denote by $\mathrm{C}$ all general constants, and $\mathrm{C}(\alpha)$ represents the dependence of constant $\mathrm{C}$ on a some parameter $\alpha$. We use $\xi$ to work in a Fourier space. 
The main aim of this paper is to use the techniques of [4] to construct solutions for the steady state MHD equations in the whole space with finite energy by imposing certain restrictions on $\mathrm{f}$. Thus, in Section II, we give preliminary concepts and develop the machinery which will be used to prove our main theorem. We also discuss about $L^{2}(\Omega)$ bounds for stationary solutions of MHD.In Section III, we prove themain existence theorem and decay of solutions. Finally, in Section IV we give concluding remarks commenting on probable future work.

We now state our main theorem. For this , as discussed above, $\stackrel{\circ}{H}_{\sigma}$ is the completion of the smooth divergence free functions of compact support. Moreover the condition $f \in X$ implies the classical assumptions $f \in L^{2}$ and $\|(\nabla U, \nabla B)\|<\infty$ i.e.it is a finite Dirichlet Integral. This is used in the statement of our Theorem.

Theorem 1. Let $M>0$ and $f \in X$ satisfies the following assumption.

(A) There exists a $\rho_{0}$ such that $\hat{\mathrm{f}}(\xi)=0$ for almost every $|\xi|<\rho_{0}$

Then there exists a constant $\mathrm{C}\left(\rho_{0}, v, \mathrm{M}\right)$ so that if $\|\mathrm{f}\|_{\mathrm{X}} \leq \mathrm{C}\left(\rho_{0}, v, \mathrm{M}\right)$ the following hold :

i) The PDE (1.2) has a weak solution $(\mathrm{U}, \mathrm{B}) \in \mathrm{H}_{\sigma}^{1} \times \mathrm{H}_{\sigma}^{1}$. It is a weak solution in the sense that for any divergence free functions of compact support $\phi, \psi$

$\langle\mathrm{U} \cdot \nabla \mathrm{U}, \phi\rangle-\langle\mathrm{B} \cdot \nabla \mathrm{U}, \phi\rangle+v\langle\nabla \mathrm{U}, \nabla \phi\rangle=\langle\mathrm{f}, \phi\rangle$

$\langle\mathrm{U} \cdot \nabla \mathrm{B}, \psi\rangle-\langle\mathrm{B} \cdot \nabla \mathrm{U}, \psi\rangle+\lambda\langle\nabla \mathrm{B}, \nabla \psi\rangle=0$

(ii) This solution satisfies $\|(\mathrm{U}, \mathrm{B})\|_{2} \leq \mathrm{M}$ and

$\|(\nabla \mathrm{U}, \nabla \mathrm{B})\|_{2} \leq \mathrm{v}^{-1}\|\mathrm{f}\|_{\mathrm{X}}$

(iii) This solution is unique among all solutions which have a finite norm \& satisfies $\|(\nabla \mathrm{U}, \nabla \mathrm{B})\|_{2} \leq \mathrm{v}^{-1}\|\mathrm{f}\|_{\mathrm{X}}$

Remark : The behavior of the constant $\mathrm{C}\left(\rho_{0}, v, \mathrm{M}\right)$ allows large $\mathrm{f}$ when the Magnetic Reynold Number is small. In this work, we assume that the Fourier transform of $\mathrm{f}$ is zero in some neighbourhood of the origin. This corresponds to exponential decay for the heat flow starting with initial data $\mathrm{f}$

\section{II.Preliminaries}

For Navier-Stokes equations, existence of weak solutions in a steady state case is well known, see for example [8-13]. Approximations with the Galerkin method \& a priori bounds along with the Banach-Alaoglu theorem helps us to construct weak solutions by finding a subsequence of approximations converging weakly to a possible solution. Then we use stronger compactness property to find the limit which is a solution of steady state equation.

For this approach we use a priori bound given by :

$$
\|(\nabla \mathrm{U}, \nabla \mathrm{B})\|_{2}^{2} \leq \mathrm{v}^{-2}\|\mathrm{f}\|_{\mathrm{X}}^{2}
$$

This is the assumptions that (U,B) has a finite Dirichlet Integral but we derive it from our assumption $f \in X$ using the estimate

$$
|\langle(f, U)\rangle| \leq\|f\|_{X}\|(\nabla U, \nabla B)\|_{2}
$$

The bound (2.1) is proved by multiplying formally (1.2A) by $\mathrm{U} \&(1.2 \mathrm{~B})$ by B respectively as follows :

$\& \quad(\mathrm{U} \cdot \nabla \mathrm{B}-\mathrm{B} \cdot \nabla \mathrm{U}-\lambda \Delta \mathrm{B}, \mathrm{B})=0$

This implies after integration by parts

$$
\langle\mathrm{U} \cdot \nabla \mathrm{U}, \mathrm{U}\rangle-\langle\mathrm{B} \cdot \nabla \mathrm{B}, \mathrm{U}\rangle+v|\nabla \mathrm{U}|^{2}-\langle\mathrm{f}, \mathrm{U}\rangle=0
$$


$\& \quad\langle\mathrm{U} \cdot \nabla \mathrm{B}, \mathrm{B}\rangle-\langle\mathrm{B} \cdot \nabla \mathrm{U}, \mathrm{B}\rangle+\lambda|\nabla \mathrm{B}|^{2}=0$

By using specific form of non-linearity $\langle\mathrm{w} \cdot \nabla \mathrm{U}, \mathrm{U}\rangle=0$ when $\nabla \cdot \mathrm{w}=0$ \& using $\langle\mathrm{B} \cdot \nabla \mathrm{B}, \mathrm{U}\rangle=-<\mathrm{B}$ $\cdot \nabla \mathrm{U}, \mathrm{B}>$ and then adding $(3.4 \mathrm{~A}) \&(3.4 \mathrm{~B})$ we get

$$
v|\nabla \mathrm{U}|^{2}+\lambda|\nabla \mathrm{B}|^{2}=\langle\mathrm{f}, \mathrm{U}\rangle
$$

Without loss of generality we assume $v<\lambda$ and henceforth we work with this condition in this chapter unless otherwise stated.

Equation (2.3) becomes

$$
v|\nabla \mathrm{U}|^{2}+v|\nabla \mathrm{B}|^{2}=\langle\mathrm{f}, \mathrm{U}\rangle
$$

On integrating (3.6) and using Poincare inequality alongwith definition of product norm, we get:

$$
v\|(\nabla \mathrm{U}, \nabla \mathrm{B})\|_{2}^{2} \leq \mathrm{C}\|\mathrm{f}\|_{\mathrm{X}}\|(\nabla \mathrm{U}, \nabla \mathrm{B})\|_{2}
$$

So, finally we get:

$$
\|(\nabla U, \nabla B)\|^{2} \leq C v^{-2}\|f\|_{X}^{2}
$$

We shall use this bound throughout our discussion. Now fix $f$ and $(\mathrm{U}, \mathrm{B})$ as a solution to $(1.2 \mathrm{~A})$ and (1.2B). ((U,B) does not depend on time). We would like to find conditions on $\mathrm{f}$ which guarantee $\|(U, B)\|_{2}<\infty$. For this, we establish "fast decay" of solution to the system:

$$
\begin{aligned}
& \mathrm{s}_{\mathrm{t}}+\mathrm{U} \cdot \nabla \mathrm{s}-\mathrm{B} \cdot \nabla \mathrm{w}+\nabla \mathrm{p}=\mathrm{v} \Delta \mathrm{s} \\
& \mathrm{w}_{\mathrm{t}}+\mathrm{U} \cdot \nabla \mathrm{w}-\mathrm{B} \cdot \nabla \mathrm{s}=\lambda \Delta \mathrm{w} \\
& \mathrm{s}(0)=\mathrm{f}, \mathrm{w}(0)=0, \nabla \cdot \mathrm{s}=0, \nabla \cdot \mathrm{w}=0
\end{aligned}
$$

Normally if $\mathrm{f}(\mathrm{s}, \mathrm{w})$ is a solution of (2.6) and then

$$
\widetilde{\mathrm{U}}=\int_{0}^{\infty} \mathrm{s}(\mathrm{t}) \mathrm{dt}
$$

$\& \quad \widetilde{\mathrm{B}}=\int_{0}^{\infty} \mathrm{w}(\mathrm{t}) \mathrm{dt}$ solves

$$
\begin{aligned}
& \mathrm{U} \cdot \nabla \widetilde{\mathrm{U}}-\mathrm{B} \cdot \nabla \widetilde{\mathrm{B}}+\nabla \mathrm{p}=\nu \Delta \widetilde{\mathrm{U}}+\mathrm{f} \\
& \mathrm{U} \cdot \nabla \widetilde{\mathrm{B}}-\mathrm{B} \cdot \nabla \widetilde{\mathrm{U}}=\lambda \Delta \widetilde{\mathrm{B}} \\
& \nabla \cdot \widetilde{\mathrm{U}}=0, \nabla \cdot \widetilde{\mathrm{B}}=0
\end{aligned}
$$

We have fixed (U,B) earlier \& it is also a solution for this PDE since it satisfies (1.2). As this PDE is linear $\& \nabla \cdot U=0, \nabla \cdot B=0$, solution is unique and thus we conclude that $\widetilde{U}=U, \& \widetilde{B}=B$

Using Minkowsky inequality for integrals, $\mathrm{L}^{2}$ decay of $\mathrm{s}$ is related to the $\mathrm{L}^{2}$ norm of $(\mathrm{U}, \mathrm{B})$ as follows

$\|\mathrm{U}\|_{2}=\left\|\int_{0}^{\infty} \mathrm{s} d \mathrm{t}\right\|_{2} \leq \int_{0}^{\infty}\|\mathrm{s}(\mathrm{t})\|_{2} \mathrm{dt}$

$\|\mathrm{B}\|_{2}=\left\|\int_{0}^{\infty} \mathrm{w} \mathrm{dt}\right\|_{2} \leq \int_{0}^{\infty}\|\mathrm{w}(\mathrm{t})\|_{2} \mathrm{dt}$

Thus, if $\|(\mathrm{s}, \mathrm{w})(\mathrm{t})\|_{2} \leq \mathrm{c}(1+\mathrm{t})^{-} \kappa$ with $\kappa>1$, then we can expect 
$(\mathrm{U}, \mathrm{B}) \in \mathrm{L}^{2} \times \mathrm{L}^{2}$.

Using a standard Fourier Splitting argument we can only get

$$
\|(U, B)\|_{2} \leq \mathrm{c}(1+\mathrm{t})^{-3 / 4}
$$

To resolve this problem we will estimate the difference:

$$
\left.\begin{array}{rl}
\ell & =\mathrm{s}-\Phi \text { where } \Phi=\mathrm{e}^{\mathrm{v} \Delta \mathrm{t}} \mathrm{f} \\
\& \quad \mathrm{~m} & =\mathrm{w}
\end{array}\right\}
$$

Here $\Phi$ is the solution to the heat equation with initial data $f$. The function $(\ell, \mathrm{m})$ satisfies a parabolic equation with zero initial data $\&$ a forcing term which can be controlled by restricting $\mathrm{f}$.

Thus using (2.8), we get our new set of equations as:

$$
\begin{gathered}
\ell_{\mathrm{t}}+\mathrm{U} \cdot \nabla \ell+\mathrm{U} \cdot \nabla \Phi-\mathrm{B} \cdot \nabla \mathrm{m}+\nabla \mathrm{P}=v \Delta \ell \\
\mathrm{m}_{\mathrm{t}}+\mathrm{U} \cdot \nabla \mathrm{m}-\mathrm{B} \cdot \nabla \ell-\mathrm{B} \cdot \nabla \Phi=\lambda \Delta \mathrm{m} \\
\Rightarrow \ell_{\mathrm{t}}+\mathrm{U} \cdot \nabla \ell-\mathrm{B} \cdot \nabla \mathrm{m}+\nabla \mathrm{P}=\nu \Delta \ell-\mathrm{U} \cdot \nabla \Phi \ldots(2.9 \mathrm{~A}) \\
\mathrm{m}_{\mathrm{t}}+\mathrm{U} \cdot \nabla \mathrm{m}-\mathrm{B} \cdot \nabla \ell=\lambda \Delta \mathrm{m}+\mathrm{B} \cdot \nabla \Phi \\
\nabla \cdot \ell=0, \quad \nabla \cdot \mathrm{m}=0 \\
(\ell, \mathrm{m})(0,0)=0
\end{gathered}
$$

Using the argument as in [4], we now make the following assumption on $\mathrm{f}$.

Assumption 1: $f \in X \&$ there exists a $\rho_{0}>0$ such that $\hat{f}(\xi)=0$ for every $|\xi|<\rho_{0}$

We now prove the following

Lemma 1 : If $\mathrm{f}$ satisfies Assumption $3.2 \& \Phi=\mathrm{e}^{\mathrm{v} \Delta \mathrm{t}}, \mathrm{f}$,

then, $\quad\|\Phi\|_{2}^{2} \leq \mathrm{e}^{-2 v \rho_{\mathrm{o}} \mathrm{t}}\|\hat{\mathrm{f}}\|_{2}^{2}$

Proof: This inequality can be easily proved by using the bound

$$
\begin{aligned}
& |\widehat{\Phi}|=\left|\mathrm{e}^{-v|\xi|^{2} \mathrm{t}} \hat{\mathrm{f}}\right| \\
\Rightarrow & |\widehat{\Phi}| \leq \mathrm{e}^{-2 v \rho_{0} \mathrm{t}}|\hat{\mathrm{f}}|
\end{aligned}
$$

and calculating the $\mathrm{L}^{2}$ norm by applying Plancherel theorem

\section{Existence theorems and decay of solutions}

Throughout this section we will assume $\mathrm{f}$ satisfies assumption $1 \&$ thus $\Phi=\mathrm{e}^{v \Delta \mathrm{t}} \mathrm{f}$ satisfies (2.10). We give attention to the study of solutions for the two auxiliary PDEs.

$$
\begin{aligned}
& \mathrm{U}^{\mathrm{i}} \cdot \nabla \mathrm{U}^{\mathrm{i}+1}-\mathrm{B}^{\mathrm{i}} \cdot \nabla \mathrm{B}^{\mathrm{i}+1}+\nabla \mathrm{p}=v \Delta \mathrm{U}^{\mathrm{i}+1}+\mathrm{f} \\
& \mathrm{U}^{\mathrm{i}} \cdot \nabla \mathrm{B}^{\mathrm{i}+1}-\mathrm{B}^{\mathrm{i}} \cdot \nabla \mathrm{U}^{\mathrm{i}+1}=\lambda \Delta \mathrm{B}^{\mathrm{i}+1} \\
& \nabla \cdot \mathrm{U}^{\mathrm{i}+1}=0, \quad \nabla \cdot \mathrm{B}^{\mathrm{i}+1}=0,
\end{aligned}
$$

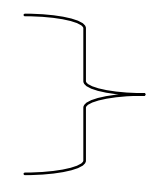


And

$$
\begin{gathered}
\ell_{\mathrm{t}}^{\mathrm{i}+1}+\mathrm{U}^{\mathrm{i}} \cdot \nabla \ell^{\mathrm{i}+1}-\mathrm{B}^{\mathrm{i}} \cdot \nabla \mathrm{m}^{\mathrm{i}+1}+\nabla \mathrm{p}=v \Delta \ell^{\mathrm{i}+1}-\mathrm{U}^{\mathrm{i}} \cdot \nabla \Phi \\
\mathrm{m}_{\mathrm{t}}^{\mathrm{i}+1}+\mathrm{U}^{\mathrm{i}} \cdot \nabla \mathrm{m}^{\mathrm{i}+1}-\mathrm{B}^{\mathrm{i}} \cdot \nabla \ell^{\mathrm{i}+1}=\lambda \Delta \mathrm{m}^{\mathrm{i}+1}+\mathrm{B}^{\mathrm{i}} \cdot \nabla \Phi \\
\nabla \cdot \ell^{\mathrm{i}+1}=0, \quad \nabla \cdot \mathrm{m}^{\mathrm{i}+1}=0, \\
\quad\left(\ell^{\mathrm{i}+1}, \mathrm{~m}^{\mathrm{i}+1}\right)(0,0)=0
\end{gathered}
$$

To deal with these partial differential equations, we take the function $\left(\mathrm{U}^{\mathrm{i}}, \mathrm{B}^{\mathrm{i}}\right) \in \mathrm{H}_{\sigma}^{1} \times \mathrm{H}_{\sigma}^{1}$ fixed before hand. Then we use these PDE's recursively to find approximate solution for (1.2) and (2.9) respectively. In what follows, we recall existence theorems for these equations and estimate the decay rate for $\left(\ell^{\mathrm{i}+1}, \mathrm{~m}^{\mathrm{i}+1}\right)$ furthermore, we make precise the meaning of

$\mathrm{U}^{\mathrm{i}}=\int_{0}^{\infty} \mathrm{s}^{\mathrm{i}}(\mathrm{t}) \mathrm{dt}$ and $\mathrm{B}^{\mathrm{i}}=\int_{0}^{\infty} \mathrm{w}^{\mathrm{i}}(\mathrm{t}) \mathrm{dt}$

then combine it with decay calculations to find uniform bounds on

$\left(\mathrm{U}^{\mathrm{i}}, \mathrm{B}^{\mathrm{i}}\right)$. Finally, we show that it is a

Cauchy sequence in $\dot{\mathrm{H}}_{\sigma}^{1} \times \dot{\mathrm{H}}_{\sigma}^{1}$ whose limit is a solution of (1.2)

We now state and prove the following existence theorems:

Theorem 2.: Let $\left(\mathrm{U}^{\mathrm{i}}, \mathrm{B}^{\mathrm{i}}\right) \in \mathrm{H}_{\sigma}^{1} \times \mathrm{H}_{\sigma}^{1}$ and $\mathrm{f} \in \mathrm{X}$ and $v<\lambda$. Then there exists a unique weak solution $\left(\mathrm{U}^{\mathrm{i}+1}, \mathrm{~B}^{\mathrm{i}+1}\right)$ to the PDE (3.1) in the sense that for any $\phi, \psi \in \mathrm{V}$

$$
\begin{aligned}
& \left\langle\mathrm{U}^{\mathrm{i}} \cdot \nabla \mathrm{U}^{\mathrm{i}+1}, \phi\right\rangle-\left\langle\mathrm{B}^{\mathrm{i}} \cdot \nabla \mathrm{B}^{\mathrm{i}+1}, \phi\right\rangle+v\left\langle\nabla \mathrm{U}^{\mathrm{i}+1}, \nabla \phi\right\rangle=\langle\mathrm{f}, \phi\rangle \\
& \left\langle\mathrm{U}^{\mathrm{i}} \cdot \nabla \mathrm{B}^{\mathrm{i}+1}, \psi\right\rangle-\left\langle\mathrm{B}^{\mathrm{i}} \cdot \nabla \mathrm{U}^{\mathrm{i}+1}, \psi\right\rangle+\lambda\left\langle\nabla \mathrm{B}^{\mathrm{i}+1}, \nabla \psi\right\rangle=0
\end{aligned}
$$

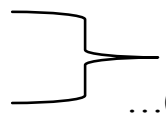

Moreover, this solution satisfies

$$
\left\|\left(\nabla U^{i+1}, \nabla B^{i+1}\right)\right\|_{2}^{2} \leq v^{-2}\|f\|_{x}^{2}
$$

Remark: The term $\nabla p$ does not appear in (3.3A) because $\nabla \phi=0$.

Proof :The procedure of the proof is by using Galerkin approximations and is well-known in the literature, see for example references [1-3]. Also, the proof can be generalized to MHD case by following the proof for Navier-Stokes equations as available in the literature ( see for example the references [8-13].

Theorem 3.: : Let $\left(\mathrm{U}^{\mathrm{i}}, \mathrm{B}^{\mathrm{i}}\right) \in \mathrm{H}_{\sigma}^{1} \times \mathrm{H}_{\sigma}^{1}$ satisfy

$$
\left\|\left(\nabla U^{i}, \nabla B^{i}\right)\right\|_{2}^{2} \leq v^{-2}\|f\|_{x}^{2}
$$

\& f satisfy the Assumption 1 with $\Phi=\mathrm{e}^{v \Delta \mathrm{t}} \mathrm{f}$ also we assume $v<\lambda$ Then there exists a unique weak solution

$$
\left(\ell^{i+1}, m^{i+1}\right) \in\left(L^{\infty}\left(R^{+}, L_{\sigma}^{2}\right) \cap L^{2}\left(R^{+}, \dot{H}_{\sigma}^{1}\right)\right) \times\left(L^{\infty}\left(R^{+}, L_{\sigma}^{2}\right) \cap L^{2}\left(R^{+}, \dot{H}_{\sigma}^{1}\right)\right)
$$

to the PDE (3.2) in the sense that for any $\phi, \psi \in \mathrm{C}^{1}\left(\mathrm{R}^{+}, \mathrm{V}\right)$ 
$\left\langle\ell_{\mathrm{t}}^{\mathrm{i}+1}, \phi\right\rangle+\left\langle\mathrm{U}^{\mathrm{i}} \cdot \nabla \ell^{\mathrm{i}+1}, \phi\right\rangle-\left\langle\mathrm{B}^{\mathrm{i}} \cdot \nabla \mathrm{m}^{\mathrm{i}+1}, \phi\right\rangle=-v\left\langle\nabla \ell^{\mathrm{i}+1}, \nabla \phi\right\rangle-\left\langle\mathrm{U}^{\mathrm{i}} \cdot \nabla \Phi, \phi\right\rangle \quad . .(3.6 \mathrm{~A})$

$\left\langle\mathrm{m}_{\mathrm{t}}^{\mathrm{i}+1}, \psi\right\rangle+\left\langle\mathrm{U}^{\mathrm{i}} \cdot \nabla \mathrm{m}^{\mathrm{i}+1}, \psi\right\rangle-<\mathrm{B}^{\mathrm{i}} \cdot \nabla \ell^{\mathrm{i}+1}, \psi>=-\lambda\left\langle\nabla \mathrm{m}^{\mathrm{i}+1}, \nabla \psi\right\rangle+<\mathrm{B}^{\mathrm{i}} \cdot \nabla \Phi, \psi>\ldots(3.6 \mathrm{~B})$

$\nabla \ell^{\mathrm{i}+1}=0, \quad \nabla \mathrm{m}^{\mathrm{i}+1}=0$

$\left(\ell^{\mathrm{i}+1}, \mathrm{~m}^{\mathrm{i}+1}\right)(0,0)=(0,0)$

Moreover this solution satisfies

$\sup _{\mathrm{t}}\left\|\left(\ell^{\mathrm{i}+1}, \mathrm{~m}^{\mathrm{i}+1}\right)(\mathrm{t})\right\|_{2}^{2}+v \int_{0}^{\infty}\left\|\left(\nabla \ell^{\mathrm{i}+1}, \nabla \mathrm{m}^{\mathrm{i}+1}\right)(\mathrm{s})\right\|_{2}^{2} \mathrm{ds} \leq \mathrm{C} \rho_{\mathrm{o}}^{-1 / 2} v^{-4}\|\mathrm{f}\|_{\mathrm{x}}^{4}$

Proof:-The partial differential equations here are closely related to MHD equations. As mentioned in the proof of previous theorem, the procedure is to construct Galerkinapproximations which satisfies a uniform estimate similar to (3.7) and then use compactness argument to pass through the limit. We now give a formal proof of (3.7) which can be used as an a priori estimate in this approach.

Multiplying (2.9A) by $\ell^{\mathrm{i}+1} \&(2.9 \mathrm{~B})$ by $\mathrm{m}^{\mathrm{i}+1}$, integrating by parts $\&$ adding and then using the bilinear relations, we get:-

$\frac{1}{2} \frac{\mathrm{d}}{\mathrm{dt}}\left\|\ell^{\mathrm{i}+1}\right\|_{2}^{2}+v\left\|\nabla \ell^{\mathrm{i}+1}\right\|^{2}+\frac{1}{2} \frac{\mathrm{d}}{\mathrm{dt}}\left\|\mathrm{m}^{\mathrm{i}+1}\right\|_{2}^{2}+\lambda\left\|\nabla \mathrm{m}^{\mathrm{i}+1}\right\|^{2}$

$=\left\langle\mathrm{U}^{\mathrm{i}} \cdot \nabla \ell^{\mathrm{i}+1}, \Phi\right\rangle+\left\langle\mathrm{B}^{\mathrm{i}} \cdot \nabla \mathrm{m}^{\mathrm{i}+1}, \Phi\right\rangle$

$\leq\left\|\mathrm{U}^{\mathrm{i}}\right\|_{6}\left\|\nabla \ell^{\mathrm{i}+1}\right\|_{2}\|\Phi\|_{3}+\left\|\mathrm{B}^{\mathrm{i}}\right\|_{6}\left\|\nabla \mathrm{m}^{\mathrm{i}+1}\right\|_{2}\|\Phi\|_{3}$

$\leq \frac{c}{v}\left\|\mathrm{U}^{\mathrm{i}}\right\|_{6}^{2}\|\Phi\|_{3}^{2}+\frac{v}{2}\left\|\nabla \ell^{\mathrm{i}+1}\right\|_{2}^{2}+\frac{c}{\lambda}\left\|\mathrm{B}^{\mathrm{i}}\right\|_{6}^{2}\|\Phi\|_{3}^{2}+\frac{\lambda}{2}\left\|\nabla \mathrm{m}^{\mathrm{i}+1}\right\|_{2}^{2}$

$$
\begin{aligned}
& \frac{1}{2} \frac{\mathrm{d}}{\mathrm{dt}}\left[\left\|\ell^{\mathrm{i}+1}\right\|_{2}^{2}+\left\|\mathrm{m}^{\mathrm{i}+1}\right\|_{2}^{2}\right]+v\left\|\nabla \ell^{\mathrm{i}+1}\right\|^{2} \lambda\left\|\nabla \mathrm{m}^{\mathrm{i}+1}\right\|^{2} \\
& \quad \leq \frac{2 c}{v}\left\|\left(\mathrm{U}^{\mathrm{i}}, \mathrm{B}^{\mathrm{i}}\right)\right\|_{6}^{2}\|\Phi\|_{3}^{2}+\frac{v}{2}\left\|\nabla \ell^{\mathrm{i}+1}\right\|_{2}^{2}+\frac{\lambda}{2}\left\|\nabla \mathrm{m}^{\mathrm{i}+1}\right\|_{2}^{2}
\end{aligned}
$$

Now, using the assumption $v<\lambda$ and the fact that

$$
\begin{aligned}
& \left\|\mathrm{U}^{\mathrm{i}}\right\|_{2} \leq\left\|\left(\mathrm{U}^{\mathrm{i}}, \mathrm{B}^{\mathrm{i}}\right)\right\|_{2}, \\
& \left\|\mathrm{~B}^{\mathrm{i}}\right\|_{2} \leq\left\|\left(\mathrm{U}^{\mathrm{i}}, \mathrm{B}^{\mathrm{i}}\right)\right\|_{2}
\end{aligned}
$$

And using the product norm $\left\|\left(\ell^{i+1}, \mathrm{~m}^{\mathrm{i}+1}\right)\right\|_{2}^{2}=\left\|\ell^{\mathrm{i}+1}\right\|_{2}^{2}+\left\|\mathrm{m}^{\mathrm{i}+1}\right\|_{2}^{2}$, our equation (3.9) becomes

$$
\begin{aligned}
& \frac{1}{2} \frac{\mathrm{d}}{\mathrm{dt}}\left\|\left(\ell^{\mathrm{i}+1}, \mathrm{~m}^{\mathrm{i}+1}\right)\right\|_{2}^{2}+\frac{v}{2}\left\|\left(\nabla \ell^{\mathrm{i}+1}, \nabla \mathrm{m}^{\mathrm{i}+1}\right)\right\|_{2}^{2} \\
& \leq\left[\frac{2 c}{v}\left\|\left(\mathrm{U}^{\mathrm{i}}, \mathrm{B}^{\mathrm{i}}\right)\right\|_{6}^{2}\|\Phi\|_{3}^{2}\right] \\
& \leq \frac{c}{v}\left\|\left(\mathrm{U}^{\mathrm{i}}, \mathrm{B}^{\mathrm{i}}\right)\right\|_{6}^{2}\|\Phi\|_{3}^{2}
\end{aligned}
$$

Here, we have used Holder's inequality and Cauchy's Inequality. Now using the Gagliardo-Nirenberg Inequality \& the assumed bound on $\left\|\left(\nabla \mathrm{U}^{\mathrm{i}}, \nabla \mathrm{B}^{\mathrm{i}}\right)\right\|_{2}^{2}$ we get:

$\frac{\mathrm{d}}{\mathrm{dt}}\left\|\left(\ell^{\mathrm{i}+1}, \mathrm{~m}^{\mathrm{i}+1}\right)\right\|_{2}^{2}+v\left\|\left(\nabla \ell^{\mathrm{i}+1}, \nabla \mathrm{m}^{\mathrm{i}+1}\right)\right\|_{2}^{2} \leq \frac{\mathrm{c}}{v^{3}}\|\mathrm{f}\|_{\mathrm{x}}^{2}\|\Phi\|_{3}^{2}$

Using Lemma 3.3, we get

$$
\int_{0}^{\mathrm{t}}\|\Phi(\mathrm{s})\|_{2}^{2} \mathrm{ds} \leq\|\mathrm{f}\|_{\mathrm{X}}^{2} \int_{0}^{\mathrm{t}} \mathrm{e}^{-2 v \rho_{0} \mathrm{~s}} \mathrm{ds} \leq \frac{\|\mathrm{ff}\|_{\mathrm{x}}^{2}}{2 v \rho_{0}}
$$

Together with the Gagliardo-Nirenberg Inequality \& the heat property 
$2 v \int_{0}^{\infty}\|\nabla \Phi(\mathrm{s})\|_{2}^{2} \mathrm{ds} \leq\|\mathrm{f}\|_{\mathrm{X}}^{2}$, we get

$$
\begin{aligned}
& \int_{0}^{\infty}\|\Phi(\mathrm{s})\|_{3}^{2} \mathrm{ds} \leq \int_{0}^{\mathrm{t}}\|\Phi(\mathrm{s})\|_{2}\|\nabla \Phi(\mathrm{s})\|_{2} \mathrm{ds} \\
& \leq\left(\int_{0}^{\mathrm{t}}\|\Phi(\mathrm{s})\|_{2}^{2} \mathrm{ds}\right)^{\frac{1}{2}}\left(\int_{0}^{\mathrm{t}}\|\Phi(\mathrm{s})\|_{2}^{2} \mathrm{ds}\right)^{\frac{1}{2}} \\
& \leq \mathrm{C}_{0}{ }^{-\frac{1}{2}} v^{-1}\|\mathrm{f}\|_{\mathrm{X}}^{2}
\end{aligned}
$$

Now integrating (3.11) in time \& then applying (3.12),we get (3.7).This completes the proof.

Remarks 3.1: In the theorem above the assumption $\left(\mathrm{U}^{\mathrm{i}}, \mathrm{B}^{\mathrm{i}}\right) \in \mathrm{H}_{\sigma}^{1} \times \mathrm{H}_{\sigma}^{1}$ is enough to ensure $\mathrm{U}^{\mathrm{i}} \cdot \nabla \mathrm{U}^{\mathrm{i}+1} \in\left(\dot{\mathrm{H}}_{\sigma}^{1}\right)^{\prime}, \mathrm{B}^{\mathrm{i}} \cdot \nabla \mathrm{B}^{\mathrm{i}+1} \in\left(\dot{\mathrm{H}}_{\sigma}^{1}\right)^{\prime}$ and $\mathrm{U}^{\mathrm{i}} \cdot \nabla \ell^{\mathrm{i}+1} \in\left(\dot{\mathrm{H}}_{\sigma}^{1}\right)^{\prime}, \mathrm{B}^{\mathrm{i}} \cdot \nabla \mathrm{m}^{\mathrm{i}+1} \in\left(\dot{\mathrm{H}}_{\sigma}^{1}\right)^{\prime}$ a.e.

That is, $\left|\left\langle\mathrm{U}^{\mathrm{i}} \cdot \nabla \mathrm{U}^{\mathrm{i}+1}, \phi\right\rangle\right| \leq \mathrm{C}\|\nabla \phi\|_{2}^{2}$

$$
\begin{aligned}
& \left|\left\langle\mathrm{B}^{\mathrm{i}} \cdot \nabla \mathrm{B}^{\mathrm{i}+1}, \phi\right\rangle\right| \leq \mathrm{C}\|\nabla \phi\|_{2}^{2} \\
& \left|<\mathrm{U}^{\mathrm{i}} \cdot \nabla \ell^{\mathrm{i}+1}, \psi>\right| \leq \mathrm{C}\|\nabla \psi\|_{2}^{2} \\
& \text { and }\left|<\mathrm{B}^{\mathrm{i}} \cdot \nabla \mathrm{m}^{\mathrm{i}+1}, \psi>\right| \leq \mathrm{C}\|\nabla \psi\|_{2}^{2}
\end{aligned}
$$

Thus multiplying the PDEs by $\mathrm{U}^{\mathrm{i}+1}, \mathrm{~B}^{\mathrm{i}+1} \& \ell^{\mathrm{i}+1}, \mathrm{~m}^{\mathrm{i}+1}$ respectively $\&$ integrating in space is justified. To see this, we choose a test function approximating either $\left(\mathrm{U}^{\mathrm{i}+1}, \mathrm{~B}^{\mathrm{i}+1}\right) \&$ pass the limit through the weak formulation (3.6). We shall use this technique in the following work.

Decay of $(l, m)$ : Here, by using the bootstrapping method \& Fourier splitting method we calculate the energy decay for $(l, m)$.Mohgaonkar and Saraykar [14 ] have derived $\mathrm{L}^{2}$ decay estimates for incompressible MHD flows. Our aim here is to find faster decay rates.For this we apply the Fourier splitting method and use the bound (3.7) to find a preliminary decay rate. This is then used to deduce a faster decay rate. We have to repeat this procedure until the lower rate is not affected by the recursion. Thus, we begin with an estimate for $|\widehat{\ell}|$ and $|\widehat{m}|$.

Lemma 2 : Let $\left(\ell^{i+1}, \mathrm{~m}^{\mathrm{i}+1}\right)$ be the solution of (3.2) given by Theorem 3 with $\left(\mathrm{U}^{\mathrm{i}}, \mathrm{B}^{\mathrm{i}}\right)$ and $\mathrm{f}$ satisfying assumptions of the same theorem. Then,

$$
\begin{aligned}
& \widehat{\ell^{\mathrm{i}+1}} \leq \mathrm{C}|\xi|\left\|\left(\mathrm{U}^{\mathrm{i}}\right)\right\|_{2}\left(\int_{\mathrm{o}}^{\mathrm{t}}\left\|\ell^{\mathrm{i}+1}\right\|_{2}(\mathrm{~s}) \mathrm{ds}+v^{-1} \rho_{\mathrm{o}}^{-1}\|\mathrm{f}\|_{\mathrm{x}}\right) \\
& \widehat{\mathrm{m}^{\mathrm{i}+1}} \leq \mathrm{C}|\zeta|\left\|\mathrm{B}^{\mathrm{i}}\right\|_{2}\left(\int_{\mathrm{o}}^{\mathrm{t}}\left\|\mathrm{m}^{\mathrm{i}+1}\right\|_{2}(\mathrm{~s}) \mathrm{ds}+v^{-1} \rho_{\mathrm{o}}^{-1}\|\mathrm{f}\|_{\mathrm{x}}\right)
\end{aligned}
$$

Proof :- Taking the Fourier Transform of differential equations in (3.2) and noting that the initial data is zero, we have

$$
\widehat{e^{\hat{i+1}}}=-\int_{0}^{t} e^{-v\left|\xi^{2}\right|(t-s)}\left(\xi \cdot \widehat{\mathrm{U}^{\mathrm{i}} \ell^{\mathrm{i}+1}}+\xi \hat{\mathrm{p}}+\xi \cdot \widehat{\mathrm{B}^{\mathrm{i}} \mathrm{m}^{\mathrm{i}+1}}+\xi \cdot \widehat{\mathrm{U}^{\mathrm{i}} \Phi}\right)(\mathrm{s}) \mathrm{ds}
$$


$\widehat{\mathrm{m}^{\mathrm{i}+1}}=-\int_{\mathrm{o}}^{\mathrm{t}} \mathrm{e}^{-\lambda\left|\zeta^{2}\right|(\mathrm{t}-\mathrm{s})}\left(\zeta \cdot \widehat{\mathrm{U}^{\mathrm{i}} \mathrm{m}^{\mathrm{i}+1}}+\zeta \cdot \widehat{\mathrm{B}^{\hat{\mathrm{i}}} \ell^{\mathrm{i}+1}}+\zeta \cdot \widehat{\mathrm{B}^{\mathrm{i}} \Phi}\right)(\mathrm{s}) \mathrm{ds}$

Now using Young's Inequality along with the Plancherel Theorem,we get

$$
\begin{gathered}
\left|\left(\widehat{\mathrm{U}^{\mathrm{i}} \ell^{\mathrm{i}+1}}\right)\right|+\left|\left(\mathrm{B}^{\widehat{\mathrm{i}} \mathrm{m}^{\mathrm{i}+1}}\right)\right|+\left|\left(\widehat{\mathrm{U}^{\mathrm{i}} \Phi}\right)\right| \leq \\
\left\|\left(\widehat{\mathrm{U}^{\mathrm{i}}, \mathrm{B}^{\mathrm{i}}}\right)\right\|_{2}\left(\left\|\ell^{\mathrm{i}+1}\right\|_{2}+\|\Phi\|_{2}+\left\|\ell^{\mathrm{i}+1}\right\|_{2}\right) \\
\left|\left(\widehat{\mathrm{U}^{\mathrm{i}} \mathrm{m}^{\mathrm{i}}+1}\right)\right|+\left|\left(\widehat{\mathrm{B}^{\mathrm{i} \ell^{\mathrm{i}+1}}}\right)\right|+\left|\left(\widehat{\mathrm{B}^{\mathrm{i}} \Phi}\right)\right| \leq \\
\left\|\left(\mathrm{U}^{\mathrm{i}}, \mathrm{B}^{\mathrm{i}}\right)\right\|_{2}\left(\|\left(\mathrm{m}^{\mathrm{i}+1}\left\|_{2}+\right\| \ell^{\mathrm{i}+1}\left\|_{2}+\right\| \Phi \|_{2}\right)\right.
\end{gathered}
$$

Now, taking the divergence of (3.14A), and then the Fourier Transform, we obtain $\left.|\hat{p}| \leq C\left(\mid \widehat{\mathrm{U}^{\mathrm{i}} \ell^{\mathrm{i}+1}}\right)|+|\left(\widehat{\mathrm{B}^{\hat{\mathrm{i}} \mathrm{m}^{\mathrm{i}+1}}}\right)|+|\left(\widehat{\mathrm{U}^{\hat{\mathrm{i}}} \Phi}\right) \mid\right)$

Using above in equation $(3.14 \mathrm{~A})$ we get

$$
\begin{aligned}
\ell^{\widehat{\mathrm{i}+1}} & \leq \mathrm{C}|\xi|\left\|\left(\mathrm{U}^{\mathrm{i}}, \mathrm{B}^{\mathrm{i}}\right)\right\|_{2} \int_{0}^{\mathrm{t}}\left(\left\|\ell^{\mathrm{i}+1}\right\|_{2}+\left\|\mathrm{m}^{\mathrm{i}+1}\right\|_{2}+\|\Phi\|_{2}\right)(\mathrm{s}) \mathrm{ds} \\
& \leq \mathrm{C}|\xi|\left\|\left(\mathrm{U}^{\mathrm{i}}, \mathrm{B}^{\mathrm{i}}\right)\right\|_{2}\left(\int_{0}^{\mathrm{t}}\left\|\ell^{\mathrm{i}+1}\right\|_{2}+\left\|\mathrm{m}^{\mathrm{i}+1}\right\|_{2}(\mathrm{~s}) \mathrm{ds}+v^{-1} \rho_{0}^{-1}\|\mathrm{f}\|_{\mathrm{x}}\right)
\end{aligned}
$$

And

$$
\begin{aligned}
& \widehat{\mathrm{m}^{\mathrm{i}+1}} \leq \mathrm{C}|\zeta|\left\|\left(\mathrm{U}^{\mathrm{i}}, \mathrm{B}^{\mathrm{i}}\right)\right\|_{2} \int_{0}^{\mathrm{t}}\left(\left\|\mathrm{m}^{\mathrm{i}+1}\right\|_{2}+\left\|\ell^{\mathrm{i}+1}\right\|_{2}+\|\Phi\|_{2}\right)(\mathrm{s}) \mathrm{ds} \\
& \leq \mathrm{C}|\zeta|\left\|\left(\mathrm{U}^{\mathrm{i}}, \mathrm{B}^{\mathrm{i}}\right)\right\|_{2}\left(\int_{0}^{\mathrm{t}}\left\|\mathrm{m}^{\mathrm{i}+1}\right\|_{2}+\left\|\ell^{\mathrm{i}+1}\right\|_{2}(\mathrm{~s}) \mathrm{ds}+\mathrm{v}^{-1} \rho_{0}^{-1}\|\mathrm{f}\|_{\mathrm{x}}\right)
\end{aligned}
$$

The last inequalities in the above estimates follow from Lemma 1

This completes the proof.

We now state a Lemma which can be proved on similar basis as Lemma 3.5 in [4].

Lemma 3 : Let $\left(\ell^{i+1}, m^{i+1}\right)$ be the solution of (3.2) given by Theorem 3 with $\left(U^{i}, B^{i}\right) \&$ f satisfying the assumptions of the same theorem.

Then, for any $\alpha, \beta \geq 4,\left(\ell^{i+1}, m^{i+1}\right)$ satisfies the differential inequality

$\frac{\mathrm{d}}{\mathrm{dt}}\left((1+\mathrm{t})^{\gamma}\left\|\left(\ell^{\mathrm{i}+1}, \mathrm{~m}^{\mathrm{i}+1}\right)\right\|_{2}^{2}\right)$

$\left.\leq \mathrm{C}\left(\gamma, \rho_{0}, v\right)\left\|\left(\mathrm{U}^{\mathrm{i}}, \mathrm{B}^{\mathrm{i}}\right)\right\|_{2}^{2}(1+\mathrm{t})^{\gamma-\frac{7}{2}}\left(\int_{0}^{\mathrm{t}}\left\|\left(\ell^{\mathrm{i}+1}, \mathrm{~m}^{\mathrm{i}+1}\right)(\mathrm{s})\right\|_{2}\right) \mathrm{ds}+\|\mathrm{f}\|_{\mathrm{X}}\right)^{2}+\mathrm{C} v^{-3}\|\mathrm{f}\|_{\mathrm{X}}^{2}\|\Phi\|_{3}^{2}(1+\mathrm{t})^{\gamma}$

Where $\gamma=\min (\alpha, \beta)$

Proof : Multiply (3.2A) by $l^{i+1} \&(3.2 \mathrm{~B})$ by $\mathrm{m}^{\mathrm{i}+1}$ then integrate by parts $\&$ then apply the bilinear relation $\&$ the assumed bound (3.5) we get. $\frac{1}{2} \frac{\mathrm{d}}{\mathrm{dt}}\left\|\ell^{\mathrm{i}+1}\right\|_{2}^{2}+v\left\|\nabla \ell^{\mathrm{i}+1}\right\|_{2}^{2}-\left\langle\mathrm{B}^{\mathrm{i}} \cdot \nabla \mathrm{m}^{\mathrm{i}+1}, \ell^{\mathrm{i}+1}\right\rangle=$ $\left\langle\mathrm{U}^{\mathrm{i}} \cdot \nabla \ell^{\mathrm{i}+1}, \phi\right\rangle \&$

$\frac{1}{2} \frac{\mathrm{d}}{\mathrm{dt}}\left\|\mathrm{m}^{\mathrm{i}+1}\right\|_{2}^{2}+\lambda\left\|\nabla \mathrm{m}^{\mathrm{i}+1}\right\|_{2}^{2}-\left\langle\mathrm{B}^{\mathrm{i}} \cdot \nabla \ell^{\mathrm{i}+1}, \mathrm{~m}^{\mathrm{i}+1}\right\rangle=\left\langle\mathrm{B}^{\mathrm{i}} \cdot \nabla \mathrm{m}^{\mathrm{i}+1}, \phi\right\rangle$

Applying same argument as in previous lemma we get from (3.11) 
$\frac{1}{2} \frac{\mathrm{d}}{\mathrm{dt}}\left\|\ell^{\mathrm{i}+1}\right\|^{2}+v\left\|\nabla \ell^{\mathrm{i}+1}\right\|^{2}$

$\leq \frac{\mathrm{C}}{v^{3}}\|\mathrm{f}\|_{\mathrm{X}}^{2}\|\Phi\|_{3}^{2}+\frac{v}{2}\left\|\nabla \ell^{\mathrm{i}+1}\right\|_{2}^{2}$

$\frac{1}{2} \frac{\mathrm{d}}{\mathrm{dt}}\left\|\mathrm{m}^{\mathrm{i}+1}\right\|^{2}+v\left\|\nabla \mathrm{m}^{\mathrm{i}+1}\right\|^{2}$

$\leq \frac{\mathrm{C}}{v^{3}}\|\mathrm{f}\|_{\mathrm{X}}^{2}\|\Phi\|_{3}^{2}+\frac{v}{2}\left\|\nabla \mathrm{m}^{\mathrm{i}+1}\right\|_{2}^{2}$

Now we split the viscous term in Fourier space around the ball $\mathrm{B}(\mathrm{R})$ using the Plancherel Theorem

$-v\left\|\nabla \ell^{\mathrm{i}+1}\right\|_{2}^{2} \leq-v \int_{\mathrm{B}(\mathrm{R}) \mathrm{C}}|\xi|\left|\ell^{\widehat{\mathrm{i}+1}}\right|{ }^{2} \mathrm{~d} \xi$

$\leq-v \mathrm{R}^{2} \int_{\mathrm{B}(\mathrm{R}) \mathrm{C}}\left|\widehat{\ell^{\mathrm{i}+1}}\right| \mathrm{d} \xi$

$\leq-v \mathrm{R}^{2}\left\|\ell^{\mathrm{i}+1}\right\|_{2}^{2}+v \mathrm{R}^{2} \int_{\mathrm{B}(\mathrm{R})}\left|\ell^{\hat{\mathrm{i}}+1}\right|^{2} \mathrm{~d} \xi$

Combining this with $(3.16 \mathrm{~A})$ we get

$\frac{\mathrm{d}}{\mathrm{dt}}\left\|\left(\ell^{\mathrm{i}+1}\right)\right\| \frac{2}{2}+v \mathrm{R}^{2}\left\|\ell^{\mathrm{i}+1}\right\| \frac{2}{2}$

$\leq v \mathrm{R}^{2} \int_{\mathrm{B}(\mathrm{R})}\left|\ell^{\widehat{\mathrm{i}+1}}\right|^{2} \mathrm{~d} \xi+\mathrm{C} v^{3}\|\mathrm{f}\|_{\mathrm{X}}^{2}\|\Phi\|_{3}^{2}$

Similarly our (3.16B) becomes

$\frac{\mathrm{d}}{\mathrm{dt}}\left\|\left(\mathrm{m}^{\mathrm{i}+1}\right)\right\| \frac{2}{2}+v \mathrm{R}^{2}\left\|\mathrm{~m}^{\mathrm{i}+1}\right\| \frac{2}{2} \leq v \mathrm{R}^{2} \int_{\mathrm{B}(\mathrm{R})}\left|\widehat{\mathrm{m}^{\mathrm{i}+1}}\right|^{2} \mathrm{~d} \zeta+\mathrm{C} v^{3}\|\mathrm{f}\|_{\mathrm{X}}^{2}\|\Phi\|_{3}^{2}$

Then using (3.13), we bound

$\int_{\mathrm{B}(\mathrm{R})}\left|\ell^{\hat{\mathrm{i}}+1}\right|^{2} \mathrm{~d} \xi \leq \mathrm{C}\left\|\mathrm{U}^{\mathrm{i}}\right\|_{2}^{2}\left(\int_{0}^{\mathrm{t}}\left\|\ell^{\mathrm{i}+1}(\mathrm{~s})\right\|_{2} \mathrm{ds}+v^{-1} \rho_{0}^{-1}\|\mathrm{f}\|_{\mathrm{X}}\right)\left(\int_{\mathrm{B}(\mathrm{R})}|\xi|^{2} \mathrm{~d} \xi\right)$

$\leq \mathrm{C}\left\|\mathrm{U}^{\mathrm{i}}\right\|_{2}^{2}\left(\int_{0}^{\mathrm{t}}\left\|\ell^{\mathrm{i}+1}\right\|_{2} \mathrm{ds}+v^{-1} \rho_{0}^{-1}\|\mathrm{f}\|_{\mathrm{X}}\right) \mathrm{R}^{5}$

Similarly,

$\int_{\mathrm{B}(\mathrm{R})}\left|\widehat{\mathrm{m}^{\mathrm{i}+1}}\right|^{2} \mathrm{~d} \xi \leq \mathrm{C}\left\|\mathrm{B}^{\mathrm{i}}\right\|_{2}^{2}\left(\int_{0}^{\mathrm{t}}\left\|\mathrm{m}^{\mathrm{i}+1}(\mathrm{~s})\right\|_{2} \mathrm{ds}+v^{-1} \rho_{0}^{-1}\|\mathrm{f}\|_{\mathrm{X}}\right)\left(\int_{\mathrm{B}(\mathrm{R})}|\zeta|^{2} \mathrm{~d} \zeta\right)$

$\leq \mathrm{C}\left\|\mathrm{B}^{\mathrm{i}}\right\|_{2}^{2}\left(\int_{0}^{\mathrm{t}}\left\|\mathrm{m}^{\mathrm{i}+1}\right\|_{2} \mathrm{ds}+\mathrm{v}^{-1} \rho_{0}^{-1}\|\mathrm{f}\|_{\mathrm{X}}\right) \mathrm{R}^{5}$

then

$\left.\frac{\mathrm{d}}{\mathrm{dt}}\left\|\left(\ell^{\mathrm{i}+1}\right)\right\| \frac{2}{2}+v \mathrm{R}^{2}\left\|\ell^{\mathrm{i}+1}\right\| \frac{2}{2} \leq \mathrm{C} \quad v \mathrm{R}^{7}\left\|\mathrm{U}^{\mathrm{i}}\right\|_{2}^{2} \quad\left(1+v^{-1} \rho_{0}^{-1}\right)^{2}\left(\int_{0}^{\mathrm{t}} \| \ell^{\mathrm{i}+1}\right)\|(\mathrm{s}) \mathrm{ds}+\| \mathrm{f} \|_{\mathrm{X}}\right)^{2}$

$+\mathrm{C} v^{-3}\|\mathrm{f}\|_{\mathrm{X}}^{2}\|\Phi\|_{3}^{2}$ 
and $\frac{\mathrm{d}}{\mathrm{dt}}\left\|\mathrm{m}^{\mathrm{i}+1}\right\| \frac{2}{2}+v \mathrm{R}^{2}\left\|\mathrm{~m}^{\mathrm{i}+1}\right\| \frac{2}{2}$

$\leq \mathrm{C} v \mathrm{R}^{7}\left\|\mathrm{~B}^{\mathrm{i}}\right\|_{2}^{2}\left(1+v^{-1} \rho_{0}^{-1}\right)^{2}\left(\int_{0}^{\mathrm{t}}\left\|\mathrm{m}^{\mathrm{i}+1}\right\|(\mathrm{s}) \mathrm{ds}+\|\mathrm{f}\|_{\mathrm{X}}\right)^{2}$

$+\mathrm{C} v^{-3}\|\mathrm{f}\|_{\mathrm{X}}^{2}\|\Phi\|_{3}^{2} \ldots(3.16 \mathrm{D})$

Here we take $R^{2}=\frac{m}{v}(1+t)^{-1}$ then use $(1+t)^{\alpha}$ as an integrating factor of $(3.16 C)$ and $(1+t)^{\beta}$ as an integrating factor of (3.16D) and subsequently adding them and using product we establish the lemma.

Theorem 4 : Let $\left(\ell^{i+1}, \mathrm{~m}^{\mathrm{i}+1}\right)$ be the solution of (2.9) given by theorem 3 with $\left(\mathrm{U}^{\mathrm{i}}, \mathrm{B}^{\mathrm{i}}\right)$ and $\mathrm{f}$ satisfying the assumptions of the same theorem. Then $\left(\ell^{\mathrm{i}+1}, \mathrm{~m}^{\mathrm{i}+1}\right)$ satisfies the decay bound

$\left\|\left(\ell^{\mathrm{i}+1}, \mathrm{~m}^{\mathrm{i}+1}\right)(\mathrm{T})\right\|_{2}^{2} \leq \mathrm{C}\left(\mu, \rho_{0}, v\right)\left(1+\left\|\left(\mathrm{U}^{\mathrm{i}}, \mathrm{B}^{\mathrm{i}}\right)\right\|_{2}^{2}\right)^{2}\left(1+\|\mathrm{f}\|_{\mathrm{X}}^{2}\right)\|\mathrm{f}\|_{\mathrm{X}}^{2}(1+\mathrm{T})^{-5 / 2}$

Where $\mu=\max ((\alpha), \beta)$

Proof : Combining the bound on $\left\|\left(\ell^{i+1}\right)\right\|_{2}$ given by (3.7) with (3.16) we write.

$\left.\frac{\mathrm{d}}{\mathrm{dt}}\left((1+\mathrm{t})^{\alpha}\left\|\left(\ell^{\mathrm{i}+1}\right)\right\|_{2}^{2}\right) \leq \mathrm{C}\left(\alpha, \rho_{0}, v\right)\left\|\left(\mathrm{U}^{\mathrm{i}}\right)\right\|_{2}^{2}\right) \quad(1+\mathrm{t})^{\alpha-7 / 2}\left(\mathrm{t}^{2}\|\mathrm{f}\|_{\mathrm{X}}^{4}+\|\mathrm{f}\|_{\mathrm{X}}^{2}\right)+\mathrm{C}(v)\|\mathrm{f}\|_{2}^{2}\|\Phi\|_{2}^{2}$ $(1+t)^{\alpha}$

$\leq \mathrm{C}\left(\alpha, \rho_{0}, v\right)\left(1+\left\|\left(\mathrm{U}^{\mathrm{i}}\right)\right\|_{2}^{2}\right)\left(1+\|\mathrm{f}\|_{\mathrm{X}}^{2}\right)\|\mathrm{f}\|_{\mathrm{X}}^{2}(1+\mathrm{t})^{\alpha-3 / 2}+\mathrm{C}(v)\|\mathrm{f}\|_{\mathrm{X}}^{2}\|\Phi\|_{3}^{2}(1+\mathrm{t})^{\alpha}$

Similarly we have

$\left.\frac{\mathrm{d}}{\mathrm{dt}}\left((1+\mathrm{t})^{\beta}\left\|\left(\mathrm{m}^{\mathrm{i}+1}\right)\right\|_{2}^{2}\right) \leq \mathrm{C}\left(\beta, \rho_{0}, v\right)\left\|\left(\mathrm{B}^{\mathrm{i}}\right)\right\|_{2}^{2}\right)(1+\mathrm{t})^{\beta-7 / 2}\left(\mathrm{t}^{2}\|\mathrm{f}\|_{\mathrm{X}}^{4}+\|\mathrm{f}\|_{\mathrm{X}}^{2}\right)+\mathrm{C}(v)\|\mathrm{f}\|_{2}^{2}\|\Phi\|_{2}^{2}$ $(1+t)^{\beta}$

$\leq \mathrm{C}\left(\beta, \rho_{0}, v\right)\left(1+\left\|\mathrm{B}^{\mathrm{i}}\right\|_{2}^{2}\right)\left(1+\|\mathrm{f}\|_{\mathrm{X}}^{2}\right)\|\mathrm{f}\|_{\mathrm{X}}^{2}(1+\mathrm{t})^{\beta-3 / 2}+\mathrm{C}(v)\|\mathrm{f}\|_{\mathrm{X}}^{2}\|\Phi\|_{3}^{2}(1+\mathrm{t})^{\beta}$

The next step is to integrate in time. The first term on the RHS of above equations can be integrated directly and the second term is estimated similar to (3.12) in each above equations. Now,

$\mathrm{C}(v)\|\mathrm{f}\|_{\mathrm{X}}^{2} \int_{0}^{\mathrm{T}}\|\Phi\|_{3}^{2}(1+\mathrm{t})^{\alpha} \mathrm{dt} \leq \mathrm{C}(\mathrm{v})\|\mathrm{f}\|_{\mathrm{X}}^{2} \int_{0}^{\mathrm{T}}\|\Phi(\mathrm{t})\|_{2}\|\nabla \Phi(\mathrm{t})\|_{2}(1+\mathrm{t})^{\alpha} \mathrm{dt}$

$\leq \mathrm{C}(v)\|\mathrm{f}\|_{\mathrm{X}}^{2}\left(\int_{0}^{\mathrm{T}}(1+\mathrm{t})^{\alpha}\|\Phi(\mathrm{t})\|_{2}^{2} \mathrm{ds}\right)^{1 / 2}\left(\int_{0}^{\mathrm{T}}\|\nabla \Phi(\mathrm{t})\|_{2}^{2}\right) \mathrm{dt}$

Similarly we have,

$\mathrm{C}(v)\|\mathrm{f}\|_{\mathrm{X}}^{2} \int_{0}^{\mathrm{T}}\|\Phi\|_{3}^{2}(1+\mathrm{t})^{\beta} \mathrm{dt} \leq \mathrm{C}(v)\|\mathrm{f}\|_{\mathrm{X}}^{2}\left(\int_{0}^{\mathrm{T}}(1+\mathrm{t})^{\beta}\|\Phi(\mathrm{t})\|_{2}^{2} \mathrm{ds}\right)^{1 / 2}\left(\int_{0}^{\mathrm{T}}\|\nabla \Phi(\mathrm{t})\|_{2}^{2}\right) \mathrm{dt}$

This gives an initial decay bound.

$\left\|\left(\ell^{\mathrm{i}+1}\right)(\mathrm{T})\right\|_{2}^{2} \leq \mathrm{C}\left(\alpha, \rho_{0}, v\right)\left(1+\left\|\mathrm{U}^{\mathrm{i}}\right\|_{2}^{2}\right)\left(1+\|\mathrm{f}\|_{\mathrm{X}}^{2}\right)\|\mathrm{f}\|_{\mathrm{X}}^{2}(1+\mathrm{T})^{-1 / 2} \ldots$
$\left\|\left(\mathrm{m}^{\mathrm{i}+1}\right)(\mathrm{T})\right\|_{2}^{2} \leq \mathrm{C}\left(\beta, \rho_{0}, v\right)\left(1+\left\|\mathrm{B}^{\mathrm{i}}\right\|_{2}^{2}\right)\left(1+\|\mathrm{f}\|_{\mathrm{X}}^{2}\right)\|\mathrm{f}\|_{\mathrm{X}}^{2}(1+\mathrm{T})^{-1 / 2}$

We now use (3.15) and (3.18) instead of (3.7) and integrate in time to obtain 
$\left\|\left(\ell^{\mathrm{i}+1}\right)(\mathrm{T})\right\|_{2}^{2} \leq \mathrm{C}\left(\alpha, \rho_{0}, v\right)\left(1+\left\|\mathrm{U}^{\mathrm{i}}\right\|_{2}^{2}\right)^{2}\left(1+\|\mathrm{f}\|_{\mathrm{X}}^{2}\right)\|\mathrm{f}\|_{\mathrm{X}}^{2}(1+\mathrm{T})^{-1}$

$\left\|\left(\mathrm{m}^{\mathrm{i}+1}\right)(\mathrm{T})\right\|_{2}^{2} \leq \mathrm{C}\left(\beta, \rho_{0}, v\right)\left(1+\left\|\mathrm{B}^{\mathrm{i}}\right\|_{2}^{2}\right)^{2}\left(1+\|\mathrm{f}\|_{\mathrm{X}}^{2}\right)\|\mathrm{f}\|_{\mathrm{X}}^{2}(1+\mathrm{T})^{-1}$

Adding above inequalities and using product norm and then following the iteration procedure six times, which gives the best decay rate, we get finally our results.

We now proceed to derive a relation between $\left(\mathrm{U}^{\mathrm{i}}, \mathrm{B}^{\mathrm{i}}\right) \&\left(\ell^{\mathrm{i}}, \mathrm{m}^{\mathrm{i}}\right)$.

Relation between $\left(\mathbf{U}^{\mathrm{i}}, \mathbf{B}^{\mathrm{i}}\right) \&\left(\ell^{\mathrm{i}}, \mathbf{m}^{\mathrm{i}}\right)$ :

We have $\mathrm{U}^{\mathrm{i}}=\int_{0}^{\infty} \mathrm{s}^{\mathrm{i}}(\mathrm{t}) \mathrm{dt}$ and $\mathrm{B}^{\mathrm{i}}=\int_{0}^{\infty} \mathrm{w}^{\mathrm{i}}(\mathrm{t}) \mathrm{dt}$. We show that approximations of these integrals are bounded uniformly in $\mathrm{L}^{2} \&$ form Cauchy sequences whose limit will be a solution of (3.1). We then apply the decay results showed above to find a uniform bound in $\mathrm{L}^{2}$ for $\left(\mathrm{U}^{\mathrm{i}}, \mathrm{B}^{\mathrm{i}}\right)$. For this we use $\Phi=$ $\mathrm{e}^{\Delta t} \mathrm{f}$. We first prove the following Lemma.

Lemma 4: Let $\left(\ell^{\mathrm{i}+1}, \mathrm{~m}^{\mathrm{i}+1}\right)$ be the solution of (2.9) given by Theorem 3 with $\left(\mathrm{U}^{\mathrm{i}}, \mathrm{B}^{\mathrm{i}}\right)$ \& $\mathrm{f}$ satisfying the assumption of the same theorem. Then the functions

$\mathrm{s}^{\mathrm{i}+1}=\ell^{\mathrm{i}+1}+\Phi \& \mathrm{w}^{\mathrm{i}+1}=\mathrm{m}^{\mathrm{i}+1}$

satisfy : $\int_{0}^{\infty} \mathrm{s}^{\mathrm{i}+1}(\mathrm{t}) \mathrm{dt} \epsilon \mathrm{L}_{\sigma}^{2} \& \int_{\mathrm{o}}^{\infty} \mathrm{w}^{\mathrm{i}+1}(\mathrm{t}) \mathrm{dt} \epsilon \mathrm{L}_{\sigma}^{2}$

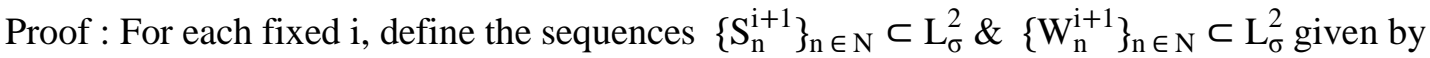

$S_{n}^{i+1}=\int_{0}^{n} s^{i+1}(t) d t$ and $W_{n}^{i+1}=\int_{0}^{n} W^{i+1}(t) d t$

Since, $s^{i+1}(t) \in L_{\sigma}^{2}$, $w^{i+1}(t) \in L_{\sigma}^{2}$ a.e. the sequences $\left\{S_{n}^{i+1}\right\} \&\left\{W_{n}^{i+1}\right\}$ are well defined. Using Minkowski's inequality for integral with assumption 1 (through (2.10) \& (3.17) the following bounds show how the sequences $\left\{\mathrm{S}_{\mathrm{n}}^{\mathrm{i}+1}\right\} \&\left\{\mathrm{~W}_{\mathrm{n}}^{\mathrm{i}+1}\right\}$ are bounded uniformally (for $\mathrm{n}$ ) in $\mathrm{L}_{\sigma}^{2}$ respectively.

$\left\|S_{n}^{i+1}\right\|_{2} \leq \int_{0}^{n}\left\|s^{i+1}(t)\right\|_{2} d t$ and $\left\|W_{n}^{i+1}\right\|_{2} \leq \int_{0}^{n}\left\|w^{i+1}(t)\right\|_{2} d t$

$\Rightarrow\left\|\mathrm{S}_{\mathrm{n}}^{\mathrm{i}+1}\right\|_{2} \leq \int_{0}^{\mathrm{n}}\left\|\ell^{\mathrm{i}+1}(\mathrm{t})\right\|_{2} \mathrm{dt}+\int_{0}^{\mathrm{n}}\|\Phi(\mathrm{t})\|_{2} \mathrm{dt} \quad$ and $\left\|\mathrm{W}_{\mathrm{n}}^{\mathrm{i}+1}\right\|_{2} \leq \int_{0}^{\mathrm{n}}\left\|\mathrm{m}^{\mathrm{i}+1}(\mathrm{t})\right\|_{2} \mathrm{dt}$

$\Rightarrow\left\|\mathrm{S}_{\mathrm{n}}^{\mathrm{i}+1}\right\|_{2} \leq \mathrm{C}\left(\rho_{0}, v\right)\left(1+\left\|\left(\mathrm{U}^{\mathrm{i}}\right)\right\|_{2}^{2}\right)^{3}\left(1+\|\mathrm{f}\|_{\mathrm{X}}^{2}\right)^{1 / 2}\|\mathrm{f}\|_{\mathrm{X}}$

and $\left\|\mathrm{W}_{\mathrm{n}}^{\mathrm{i}+1}\right\|_{2} \leq \mathrm{C}\left(\rho_{0}, v\right)\left(1+\left\|\mathrm{B}^{\mathrm{i}}\right\|_{2}^{2}\right)^{3}\left(1+\|\mathrm{f}\|_{\mathrm{X}}^{2}\right)^{1 / 2}\|\mathrm{f}\|_{\mathrm{X}}$

$\left\|\left(\mathrm{S}_{\mathrm{n}}^{\mathrm{i}+1}, \mathrm{~W}_{\mathrm{n}}^{\mathrm{i}+1}\right)\right\|_{2} \leq \int_{\mathrm{o}}^{\mathrm{n}}\left\|\left(\ell^{\mathrm{i}+1}, \mathrm{~m}^{\mathrm{i}+1}\right)(\mathrm{t})\right\|_{2} \mathrm{dt}+\int_{\mathrm{o}}^{\mathrm{n}}\|\Phi(\mathrm{t})\|_{2} \mathrm{dt}$

$\leq \mathrm{C}\left(\rho_{0}, v, \lambda\right)\left(1+\left\|\left(\mathrm{U}^{\mathrm{i}}, \mathrm{B}^{\mathrm{i}}\right)\right\|_{2}^{2}\right)^{3}\left(1+\|\mathrm{f}\|_{\mathrm{X}}^{2}\right)^{1 / 2}\|\mathrm{f}\|_{\mathrm{X}}$

Similarly, we can have:

$$
\left\|S_{n+1}^{\mathrm{i}+1}-S_{n}^{\mathrm{i}+1}\right\|_{2} \leq \int_{\mathrm{n}}^{\mathrm{n}+1}\left\|\left(\mathrm{~s}^{\mathrm{i}+1}\right)\right\|_{2} \mathrm{dt}
$$


$\left\|\mathrm{W}_{\mathrm{n}+1}^{\mathrm{i}+1}-\mathrm{W}_{\mathrm{n}}^{\mathrm{i}+1}\right\|_{2} \leq \int_{\mathrm{n}}^{\mathrm{n}+1}\left\|\left(\mathrm{w}^{\mathrm{i}+1}\right)\right\|_{2} \mathrm{dt}$.

Observing (3.17) \& the decay of $\Phi$ implied by assumption 2.1 we know that integrals $\int_{\mathrm{o}}^{\infty}\left\|\mathrm{s}^{\mathrm{i}+1}\right\|_{2} \mathrm{dt}$ and $\int_{\mathrm{o}}^{\infty}\left\|\mathrm{w}^{\mathrm{i}+1}\right\|_{2} \mathrm{dt}$ are finite. Hence the R.H.S of (3.19A) and (3.19B) tends to zero as $\mathrm{n}$ $\rightarrow \infty$. Following a well known argument to prove a contraction lemma we conclude that $\left\{S_{n}^{i+1}\right\}_{n \in N}$ and $\left\{\left(\mathrm{W}_{\mathrm{n}}^{\mathrm{i}+1}\right)\right\}_{\mathrm{n} \in \mathrm{N}}$ are Cauchy sequences in $\mathrm{L}_{\sigma}^{2} \&$ have a limit which we denote by $\int_{0}^{\infty} \mathrm{s}^{\mathrm{i}+1}(\mathrm{t}) \mathrm{dt}$ and $\int_{0}^{\infty} w^{i+1}(t) d t$ respectively

Remark 2: The above lemma also implies that $\int_{0}^{\infty} \mathrm{s}^{\mathrm{i}+1}(\mathrm{t}) \mathrm{dt}$ and $\int_{0}^{\infty} \mathrm{w}^{\mathrm{i}+1}(\mathrm{t}) \mathrm{dt}$ are both finite a.e. in $\mathrm{R}^{3}$

Furthermore we prove the following .

Lemma 5 : Let $\left(\ell^{i+1}, \mathrm{~m}^{\mathrm{i}+1}\right)$ be the solution of (2.9) given by the Theorem 3 with $\left(\mathrm{U}^{\mathrm{i}}, \mathrm{B}^{\mathrm{i}}\right) \& \mathrm{f}$ satisfying the assumption of the theorem. The functions $\mathrm{s}^{\mathrm{i}+1}=\ell^{\mathrm{i}+1}+\Phi \& \mathrm{w}^{\mathrm{i}+1}=\mathrm{m}^{\mathrm{i}+1}$ satisfy

$$
\int_{0}^{\infty} \mathrm{s}^{\mathrm{i}+1}(\mathrm{t}) \mathrm{dt}=\mathrm{U}^{\mathrm{i}+1} \text { and } \int_{0}^{\infty} \mathrm{w}^{\mathrm{i}+1}(\mathrm{t}) \mathrm{dt}=\mathrm{B}^{\mathrm{i}+1}
$$

Proof : : To prove this lemma we show $\left(\int_{0}^{\infty} \mathrm{s}^{\mathrm{i}+1}(\mathrm{t}) \mathrm{dt}, \int_{0}^{\infty} \mathrm{w}^{\mathrm{i}+1}(\mathrm{t}) \mathrm{dt}\right)$ is a weak solution for (3.1) to conclude the desired result. Let $\left\{\left(\mathrm{S}_{\mathrm{n}}^{\mathrm{i}+1}\right)\right\}_{\mathrm{n} \in \mathrm{N}}$ and $\left\{\left(\mathrm{W}_{\mathrm{n}}^{\mathrm{i}+1}\right)\right\}_{\mathrm{n} \in \mathrm{N}}$ be as in the previous proof.

In (3.6) choose $\varphi$ and $\psi$ to be any member of $V$ (so that it is constant in time) use the relation $\mathrm{s}^{\mathrm{i}+1}=$ $\ell^{i+1}+\Phi \& \mathrm{w}^{\mathrm{i}+1}=\mathrm{m}^{\mathrm{i}+1}$ then integrate in time.

$\int_{0}^{\mathrm{n}}\left(\frac{\mathrm{d}}{\mathrm{dt}}\left\langle\mathrm{s}^{\mathrm{i}+1}(\mathrm{t}), \varphi\right\rangle+\left\langle\mathrm{U}^{\mathrm{i}} \cdot \nabla \mathrm{s}^{\mathrm{i}+1}, \varphi\right\rangle-\left\langle\mathrm{B}^{\mathrm{i}} \cdot \nabla \mathrm{w}^{\mathrm{i}+1}, \varphi\right\rangle\right)$

$=-v \int_{0}^{\mathrm{n}}\left\langle\left(\nabla \mathrm{s}^{\mathrm{i}+1}(\mathrm{t}), \nabla \varphi\right\rangle \&\right.$

$\int_{0}^{\mathrm{n}}\left(\frac{\mathrm{d}}{\mathrm{dt}}\left\langle\mathrm{w}^{\mathrm{i}+1}, \psi\right\rangle+\left\langle\mathrm{U}^{\mathrm{i}} \cdot \nabla \mathrm{w}^{\mathrm{i}+1}, \psi\right\rangle-\left\langle\mathrm{B}^{\mathrm{i}} \cdot \nabla \mathrm{s}^{\mathrm{i}+1}, \psi\right\rangle\right)$

$=-\lambda \int_{0}^{\mathrm{n}}\left\langle\left(\nabla \mathrm{w}^{\mathrm{i}+1}, \nabla \psi\right\rangle \ldots(3.20 \mathrm{~B})\right.$

After changing the order of integration \& evaluation the first integral the become

$\left.\left\langle\mathrm{s}^{\mathrm{i}+1}(\mathrm{n}), \varphi\right\rangle+\left\langle\mathrm{U}^{\mathrm{i}} \cdot \nabla \mathrm{S}_{\mathrm{n}}^{\mathrm{i}+1}, \varphi\right\rangle-\left\langle\mathrm{B}^{\mathrm{i}} \cdot \nabla \mathrm{W}_{\mathrm{n}}^{\mathrm{i}+1}, \varphi\right\rangle\right)=-v\left\langle\nabla \mathrm{S}_{\mathrm{n}}^{\mathrm{i}+1}, \nabla \varphi\right\rangle+\langle\mathrm{f}, \varphi\rangle \&$

$\left.\left\langle\mathrm{w}^{\mathrm{i}+1}(\mathrm{n}), \psi\right\rangle+\left\langle\mathrm{U}^{\mathrm{i}} \cdot \nabla \mathrm{W}_{\mathrm{n}}^{\mathrm{i}+1}, \psi\right\rangle-\left\langle\mathrm{B}^{\mathrm{i}} \cdot \nabla \mathrm{S}_{\mathrm{n}}^{\mathrm{i}+1}, \psi\right\rangle\right)=-\lambda\left\langle\nabla \mathrm{W}_{\mathrm{n}}^{\mathrm{i}+1}, \nabla \psi\right\rangle$

Observe the first term on the LHS tends to zero as $n \rightarrow \infty$ of both the above equations. This follows form the decay bound (3.16)

$$
\begin{aligned}
& \left|\left\langle\mathrm{U}^{\mathrm{i}} \cdot \nabla\left(\mathrm{S}_{\mathrm{n}}^{\mathrm{i}+1}-\mathrm{S}^{\mathrm{i}+1}\right), \varphi\right\rangle\right| \leq \mathrm{C}\left\|\nabla \mathrm{U}^{\mathrm{i}}\right\|_{2}\left\|\left(\mathrm{~S}_{\mathrm{n}}^{\mathrm{i}+1}-\mathrm{S}^{\mathrm{i}+1}\right)\right\|_{2}\|\nabla \varphi\|_{3} \\
& \leq \mathrm{C}\left\|\left(\nabla \mathrm{U}^{\mathrm{i}}, \nabla \mathrm{B}^{\mathrm{i}}\right)\right\|_{2}\left\|\left(\mathrm{~S}_{\mathrm{n}}^{\mathrm{i}+1}-\mathrm{S}^{\mathrm{i}+1}\right)\right\|_{2}\|\nabla \varphi\|_{3}
\end{aligned}
$$

Similarly 


$$
\begin{aligned}
& \left|\left\langle\mathrm{B}^{\mathrm{i}} \cdot \nabla\left(\mathrm{W}_{\mathrm{n}}^{\mathrm{i}+1}-\mathrm{W}^{\mathrm{i}+1}\right), \psi\right\rangle\right| \leq \mathrm{C}\left\|\nabla \mathrm{B}^{\mathrm{i}}\right\|_{2}\left\|\left(\mathrm{~W}_{\mathrm{n}}^{\mathrm{i}+1}-\mathrm{W}^{\mathrm{i}+1}\right)\right\|_{2}\|\nabla \psi\|_{3} \\
& \leq \mathrm{C}\left\|\nabla \mathrm{B}^{\mathrm{i}}\right\|_{2}\left\|\left(\mathrm{~W}_{\mathrm{n}}^{\mathrm{i}+1}-\mathrm{W}^{\mathrm{i}+1}\right)\right\|_{2}\|\nabla \psi\|_{3}
\end{aligned}
$$

As $\mathrm{n} \rightarrow \infty$ this tends to zero for each test function $\varphi$ belong to $\mathrm{V}$, Hence $\left(\mathrm{S}^{\mathrm{i}+1}, \mathrm{~W}^{\mathrm{i}+1}\right)$ is a weak solution of (3.1). The uniqueness implied by Theorem 2 finishes the proof of the lemma.

Lemma 6 : Let $\left(U^{i+1}, B^{i+1}\right)$ be the solution of (1.2) given by Theorem 2 with $\left(U^{i}, B^{i}\right) \&$ satisfying Assumption of the theorem. Then the function $\left(\mathrm{U}^{\mathrm{i}+1}, \mathrm{~B}^{\mathrm{i+1}}\right)$ satisfies

$$
\left\|\left(\mathrm{U}^{\mathrm{i}+1}, \mathrm{~B}^{\mathrm{i}+1}\right)\right\|_{2} \leq \mathrm{C}\left(\rho_{0}, v\right)\left(1+\left\|\left(\mathrm{U}^{\mathrm{i}}, \mathrm{B}^{\mathrm{i}}\right)\right\|_{2}^{2}\right)^{3}\left(1+\|\mathrm{f}\|_{\mathrm{X}}^{2}\right)^{1 / 2}\|\mathrm{f}\|_{\mathrm{X}}
$$

Remark 3: The constant $\mathrm{C}\left(\rho_{0}, v\right)$ in the above Theorem tends to $\infty$ as $\rho_{0} \rightarrow 0$ or $v \rightarrow 0$ It tends to 0 as $v \rightarrow \infty$ (See Proof of Lemma3)

Proof : Define $\mathrm{s}^{\mathrm{i}+1}=\ell^{\mathrm{i}+1}+\Phi \& \quad \mathrm{w}^{\mathrm{i}+1}=\mathrm{m}^{\mathrm{i}+1}$ Just as in the proof of Lemma 5 combine Minkowski's inequality for integral (2.5) \& (3.13) but this time use the relation from Lemma 6

$$
\begin{aligned}
& \left\|\mathrm{U}^{\mathrm{i}+1}\right\|_{2} \leq \int_{0}^{\mathrm{n}}\left\|\mathrm{s}^{\mathrm{i}+1}(\mathrm{t})\right\|_{2} \mathrm{dt} \\
& \leq \int_{0}^{\mathrm{n}}\left\|\ell^{\mathrm{i}+1}(\mathrm{t})\right\|_{2} \mathrm{dt}+\int_{0}^{\mathrm{n}}\|\Phi(\mathrm{t})\|_{2} \mathrm{dt} \quad \&
\end{aligned}
$$$$
\left\|\mathrm{B}^{\mathrm{i}+1}\right\|_{2}
$$

$\leq \int_{0}^{\mathrm{n}}\left\|\mathrm{w}^{\mathrm{i}+1}(\mathrm{t})\right\|_{2} \mathrm{dt} \leq \int_{0}^{\mathrm{n}}\left\|\mathrm{m}^{\mathrm{i}+1}(\mathrm{t})\right\|_{2} \mathrm{dt}$

$\Rightarrow\left\|\mathrm{U}^{\mathrm{i}+1}\right\|_{2} \leq \mathrm{C}\left(\rho_{0}, v\right)\left(1+\left\|\mathrm{U}^{\mathrm{i}}\right\|_{2}^{2}\right)^{3}\left(1+\|\mathrm{f}\|_{\mathrm{X}}^{2}\right)^{1 / 2}\|\mathrm{f}\|_{\mathrm{X}}$

and $\left\|B^{i+1}\right\|_{2} \leq C\left(\rho_{0}, v\right)\left(1+\left\|B^{i}\right\|_{2}^{2}\right)^{3}\left(1+\|f\|_{X}^{2}\right)^{1 / 2}\|f\|_{X}$

Adding above inequalities and using product norm we get our final result.

Convergence of $\left(\mathrm{U}^{\mathrm{i}}, \mathrm{B}^{\mathrm{i}}\right)$ : We now find the limit of the approximating sequence $\left(\mathrm{U}^{\mathrm{i}}, \mathrm{B}^{\mathrm{i}}\right)$ and show this is a solution of the steady state MHD equation. We first prove :

Lemma 7 Let $\left(\mathrm{U}^{\mathrm{i}}, \mathrm{B}^{\mathrm{i}}\right)$ be the solution of (1.2) given by Theorem 2 with $\left(\mathrm{U}^{\mathrm{i}}, \mathrm{B}^{\mathrm{i}}\right)$ \& satisfies the assumption of the theorem. There exists a constant $\mathrm{C}\left(\rho_{0}, v\right)$ so that if $\|\mathrm{f}\|_{\mathrm{X}}^{2}<\mathrm{C}\left(\rho_{0}, v, \mathrm{M}\right)$ then $\left\|\left(\mathrm{U}^{\mathrm{i}}, \mathrm{B}^{\mathrm{i}}\right)\right\|_{2} \leq \mathrm{M}$.

Proof : By setting the RHS of (3.21A) and (3.21B) equal to $\mathrm{M}^{2} \&$ considering $\mathrm{Z}=\|\mathrm{f}\|_{\mathrm{X}}^{2}$ as a variable the proof is reduced to finding proof of the polynomial as below

$\mathrm{Z}^{2}+\mathrm{Z}=\mathrm{L}=\frac{\mathrm{M}^{2}}{\mathrm{C}\left(\rho_{0}, v\right)\left(1+\mathrm{M}^{2}\right)^{6}}$

Here $C\left(\rho_{0}, v\right)$ is exactly as in (3.22) since $L>0$. This Polynomial always has a strictly positive root, in this case the root is exactly the constant in the statement of the lemma. Indeed, 
$\frac{-1+\sqrt{1+4 \mathrm{~L}}}{2} \leq \sqrt{\mathrm{L}}=\frac{\mathrm{M}}{\sqrt{\mathrm{C}\left(\rho_{0}, v, \lambda\right)}\left(1+\mathrm{M}^{2}\right)^{3}}$

Theorem 5: Let $M>0$ and $f$ satisfy Assumption 1.Then there exists a constant $C\left(\rho_{o}, \nu, M\right)$ such that if $\|\mathrm{f}\|_{\mathrm{X}} \leq \mathrm{C}\left(\rho_{\mathrm{o}}, v, \mathrm{M}\right)$, the following hold:

(i)The PDE (1.2) has a weak solution $(\mathrm{U}, \mathrm{B}) \in \mathrm{H}_{\sigma}^{1} \times \mathrm{H}_{\sigma}^{1}$

(ii)This solution satisfies $\|(\mathrm{U}, \mathrm{B})\|_{2} \leq \mathrm{M}$ in addition to (2.1) and this solution is unique among all solutions which satisfy (2.1) \& have a finite $\mathrm{L}_{\sigma}^{2}$ norm.

Proof : Chose $\left(\mathrm{U}^{0}, \mathrm{~B}^{0}\right) \in \mathrm{H}_{\sigma}^{1} \times \mathrm{H}_{\sigma}^{1}$ so that $\left\|\left(\mathrm{U}^{0}, \mathrm{~B}^{0}\right)\right\|_{2} \leq \mathrm{M} \&\left\|\left(\nabla \mathrm{U}^{0}, \nabla \mathrm{B}^{0}\right)\right\|_{2} \leq v^{-2}\|\mathrm{f}\|_{\mathrm{X}}^{2}$. To construct such a function one could fix $\mathrm{f}$ and then take a solution (U,B) for (1.2). However a priori , the solution is not known to be unique or have finite $\mathrm{L}_{\sigma}^{2}$ norm. Following the procedure as mentioned in the proof of Theorem 3.17 in Bjorland and Schonbek [04] it is possible to limit $\mathrm{L}_{\sigma}^{2} \times \mathrm{L}_{\sigma}^{2}$ norm without increasing the $\dot{\mathrm{H}}_{\sigma}^{1} \times \dot{\mathrm{H}}_{\sigma}^{1}$ norm. Starting with $\left(\mathrm{U}^{0}, \mathrm{~B}^{0}\right)$ we solve (3.1) recursively using Theorem 2 to find a sequence $\{(\mathrm{Ui}, \mathrm{Bi})\}_{i=0}^{\infty}$. which satisfies $\left\|\left(\nabla \mathrm{U}^{\mathrm{i}}, \nabla \mathrm{B}^{\mathrm{i}}\right)\right\|_{2} \leq v^{-2}\|\mathrm{f}\|_{\mathrm{x}}^{2}$. Then Lemma 6 gives the uniform bound $\left\|\left(\mathrm{U}^{\mathrm{i}}, \mathrm{B}^{\mathrm{i}}\right)\right\|_{2} \leq \mathrm{M}$. Hence, its limit, if it exists must also satisfy this bound. We now show that this sequence is Cauchy in $\dot{\mathrm{H}}_{\sigma}^{1} \times \dot{\mathrm{H}}_{\sigma}^{1}$ \& its limit exists.

The difference $\mathrm{Y}^{\mathrm{i}+1}=\mathrm{U}^{\mathrm{i}+1}-\mathrm{U}^{\mathrm{i}}$ and $\mathrm{Z}^{\mathrm{i}+1}=\mathrm{B}^{\mathrm{i}+1}-\mathrm{B}^{\mathrm{i}}$ satisfies the differential equation

$$
\mathrm{U}^{\mathrm{i}} \cdot \nabla \mathrm{Y}^{\mathrm{i}+1}+\mathrm{Y}^{\mathrm{i}} \cdot \nabla \mathrm{U}^{\mathrm{i}}-\mathrm{B}^{\mathrm{i}} \cdot \nabla \mathrm{Z}^{\mathrm{i}+1}-\mathrm{Z}^{\mathrm{i}} \cdot \nabla \mathrm{B}^{\mathrm{i}}=v \Delta \mathrm{Y}^{\mathrm{i}+1}
$$

$\& \quad \mathrm{U}^{\mathrm{i}} \cdot \nabla \mathrm{Z}^{\mathrm{i}+1}+\mathrm{Y}^{\mathrm{i}} \cdot \nabla \mathrm{B}^{\mathrm{i}}-\mathrm{B}^{\mathrm{i}} \cdot \nabla \mathrm{Y}^{\mathrm{i}+1}-\mathrm{Z}^{\mathrm{i}} \cdot \nabla \mathrm{U}^{\mathrm{i}}=\lambda \Delta \mathrm{Z}^{\mathrm{i}+1}$

Multiply equation (3.36A) by $\mathrm{Y}^{\mathrm{i}+1}$ and equation (3.36B) by $\mathrm{Z}^{\mathrm{i}+1}$ respectively we get

$$
\begin{array}{r}
\left\langle\mathrm{Y}^{\mathrm{i}} \cdot \nabla \mathrm{Y}^{\mathrm{i}+1}, \mathrm{U}^{\mathrm{i}}\right\rangle-\left\langle\mathrm{B}^{\mathrm{i}} \cdot \nabla \mathrm{Z}^{\mathrm{i}+1}, \mathrm{Y}^{\mathrm{i}+1}\right\rangle+\left\langle\mathrm{Z}^{\mathrm{i}} \cdot \nabla \mathrm{Y}^{\mathrm{i}+1}, \mathrm{~B}^{\mathrm{i}}\right\rangle=v\left\|\nabla \mathrm{Y}^{\mathrm{i}+1}\right\|_{2}^{2} \\
\& \quad\left\langle\mathrm{Y}^{\mathrm{i}} \cdot \nabla \mathrm{Z}^{\mathrm{i}+1}, \mathrm{~B}^{\mathrm{i}}\right\rangle-\left\langle\mathrm{B}^{\mathrm{i}} \cdot \nabla \mathrm{Z}^{\mathrm{i}+1}, \mathrm{Y}^{\mathrm{i}+1}\right\rangle+\left\langle\mathrm{Z}^{\mathrm{i}} \cdot \nabla \mathrm{Z}^{\mathrm{i}+1}, \mathrm{U}^{\mathrm{i}}\right\rangle=\lambda\left\|\nabla \mathrm{Z}^{\mathrm{i}+1}\right\|_{2}^{2}
\end{array}
$$

Then integrating \& adding (3.24A) and (3.24B) and using bilinear relation (2.2) we get

$$
\begin{aligned}
& v\left\|\nabla Y^{i+1}\right\|_{2}^{2}+\lambda\left\|\nabla Z^{i+1}\right\|_{2}^{2} \leq\left\|Y^{i}\right\|_{6}\left\|\nabla Y^{i+1}\right\|_{2}\left\|U^{i}\right\|_{3} \\
& +\left\|Z^{\mathrm{i}}\right\|_{6}\left\|\nabla \mathrm{Y}^{\mathrm{i}+1}\right\|_{2}\left\|\mathrm{~B}^{\mathrm{i}}\right\|_{3}+ \\
& +\quad\left\|\mathrm{Y}^{\mathrm{i}}\right\|_{6}\left\|\nabla \mathrm{Z}^{\mathrm{i}+1}\right\|_{2}\left\|\mathrm{~B}^{\mathrm{i}}\right\|_{3} \\
& +\quad\left\|Z^{\mathrm{i}}\right\|_{6}\left\|\nabla \mathrm{Z}^{\mathrm{i}+1}\right\|_{2}\left\|\mathrm{U}^{\mathrm{i}}\right\|_{3} \\
& \leq \quad\left\|\nabla \mathrm{Y}^{\mathrm{i}}\right\|_{2}\left\|\nabla \mathrm{Y}^{\mathrm{i}+1}\right\|_{2}\left\|\nabla \mathrm{U}^{\mathrm{i}}\right\|_{2} \\
& +\quad\left\|\nabla Z^{\mathrm{i}}\right\|_{2}\left\|\nabla \mathrm{Y}^{\mathrm{i}+1}\right\|_{2}\left\|\nabla \mathrm{B}^{\mathrm{i}}\right\|_{2} \\
& +\quad\left\|\nabla \mathrm{Y}^{\mathrm{i}}\right\|_{2}\left\|\nabla \mathrm{Z}^{\mathrm{i}+1}\right\|_{2}\left\|\nabla \mathrm{B}^{\mathrm{i}}\right\|_{2} \\
& +\quad\left\|\nabla Z^{\mathrm{i}}\right\|_{2}\left\|\nabla \mathrm{Z}^{\mathrm{i}+1}\right\|_{2}\left\|\nabla \mathrm{U}^{\mathrm{i}}\right\|_{2} \\
& \leq \quad\left\|\left(\nabla \mathrm{Y}^{\mathrm{i}}, \nabla \mathrm{Z}^{\mathrm{i}}\right)\right\|_{2}\left\|\left(\nabla \mathrm{U}^{\mathrm{i}}, \nabla \mathrm{B}^{\mathrm{i}}\right)\right\|_{2}\left\|\nabla \mathrm{Y}^{\mathrm{i}+1}\right\|_{2} \\
& +\quad\left\|\left(\nabla \mathrm{Y}^{\mathrm{i}}, \nabla \mathrm{Z}^{\mathrm{i}}\right)\right\|_{2}\left\|\left(\nabla \mathrm{U}^{\mathrm{i}}, \nabla \mathrm{B}^{\mathrm{i}}\right)\right\|_{2}\left\|\nabla \mathrm{Y}^{\mathrm{i}+1}\right\|_{2} \\
& +\quad\left\|\left(\nabla \mathrm{Y}^{\mathrm{i}}, \nabla \mathrm{Z}^{\mathrm{i}}\right)\right\|_{2}\left\|\left(\nabla \mathrm{U}^{\mathrm{i}}, \nabla \mathrm{B}^{\mathrm{i}}\right)\right\|_{2}\left\|\nabla \mathrm{Z}^{\mathrm{i}+1}\right\|_{2} \\
& +\quad\left\|\left(\nabla \mathrm{Y}^{\mathrm{i}}, \nabla \mathrm{Z}^{\mathrm{i}}\right)\right\|_{2}\left\|\left(\nabla \mathrm{U}^{\mathrm{i}}, \nabla \mathrm{B}^{\mathrm{i}}\right)\right\|_{2}\left\|\nabla \mathrm{Z}^{\mathrm{i}+1}\right\|_{2}
\end{aligned}
$$




$$
\begin{array}{ll}
\leq \quad & 2\left\|\left(\nabla \mathrm{Y}^{\mathrm{i}}, \nabla \mathrm{Z}^{\mathrm{i}}\right)\right\|_{2}\left\|\left(\nabla \mathrm{U}^{\mathrm{i}}, \nabla \mathrm{B}^{\mathrm{i}}\right)\right\|_{2}\left\|\nabla \mathrm{Y}^{\mathrm{i}+1}\right\|_{2} \\
& +2\left\|\left(\nabla \mathrm{Y}^{\mathrm{i}}, \nabla \mathrm{Z}^{\mathrm{i}}\right)\right\|_{2}\left\|\left(\nabla \mathrm{U}^{\mathrm{i}}, \nabla \mathrm{B}^{\mathrm{i}}\right)\right\|_{2}\left\|\nabla \mathrm{Z}^{\mathrm{i}+1}\right\|_{2} \\
\leq & \frac{2 c_{1}}{v}\left\|\left(\nabla \mathrm{Y}^{\mathrm{i}}, \nabla \mathrm{Z}^{\mathrm{i}}\right)\right\|_{2}^{2}\left\|\left(\nabla \mathrm{U}^{\mathrm{i}}, \nabla \mathrm{B}^{\mathrm{i}}\right)\right\|_{2}^{2}+\frac{v}{2}\left\|\nabla \mathrm{Y}^{\mathrm{i}+1}\right\|_{2}^{2} \\
+ & \frac{2 c_{1}}{\lambda}\left\|\left(\nabla \mathrm{Y}^{\mathrm{i}}, \nabla \mathrm{Z}^{\mathrm{i}}\right)\right\|_{2}^{2}\left\|\left(\nabla \mathrm{U}^{\mathrm{i}}, \nabla \mathrm{B}^{\mathrm{i}}\right)\right\|_{2}^{2}+\frac{\lambda}{2}\left\|\nabla \mathrm{Z}^{\mathrm{i}+1}\right\|_{2}^{2}+
\end{array}
$$

Hence,

$\frac{v}{2}\left\|\nabla \mathrm{Y}^{\mathrm{i}+1}\right\|_{2}^{2}+\frac{\lambda}{2}\left\|\nabla \mathrm{Z}^{\mathrm{i}+1}\right\|_{2}^{2} \leq \frac{c}{v}\left\|\left(\nabla \mathrm{Y}^{\mathrm{i}}, \nabla \mathrm{Z}^{\mathrm{i}}\right)\right\|_{2}^{2}\left\|\left(\nabla \mathrm{U}^{\mathrm{i}}, \nabla \mathrm{B}^{\mathrm{i}}\right)\right\|_{2}^{2}$

using $v<\lambda$ and standard product norm in (3.25)we get

$$
\begin{aligned}
\frac{v}{2}\left\|\left(\nabla \mathrm{Y}^{\mathrm{i}+1}, \nabla \mathrm{Z}^{\mathrm{i}+1}\right)\right\|_{2}^{2} & \leq \frac{c}{v}\left\|\left(\nabla \mathrm{Y}^{\mathrm{i}}, \nabla \mathrm{Z}^{\mathrm{i}}\right)\right\|_{2}^{2}\left\|\left(\nabla \mathrm{U}^{\mathrm{i}}, \nabla \mathrm{B}^{\mathrm{i}}\right)\right\|_{2}^{2} \\
\left\|\left(\nabla \mathrm{Y}^{\mathrm{i}+1}, \nabla \mathrm{Z}^{\mathrm{i}+1}\right)\right\|_{2}^{2} \leq & 2 \mathrm{C} v^{-2}\left\|\left(\nabla \mathrm{Y}^{\mathrm{i}}, \nabla \mathrm{Z}^{\mathrm{i}}\right)\right\|_{2}^{2}\left\|\left(\nabla \mathrm{U}^{\mathrm{i}}, \nabla \mathrm{B}^{\mathrm{i}}\right)\right\|_{2}^{2} \\
& \leq 2 \mathrm{C} v^{-3} \mathrm{M}\|\mathrm{f}\|_{\mathrm{x}}\left\|\left(\nabla \mathrm{Y}^{\mathrm{i}}, \nabla \mathrm{Z}^{\mathrm{i}}\right)\right\|_{2}^{2} \ldots .(3
\end{aligned}
$$

We note that multiplication by $\mathrm{Y}^{\mathrm{i}+1} \& \mathrm{Z}^{\mathrm{i}+1}$ is justified since all $\mathrm{U}^{\mathrm{i}}, \mathrm{B}^{\mathrm{i}}$ and hence all $\mathrm{Y}^{\mathrm{i}}, \mathrm{Z}^{\mathrm{i}}$ are bounded in $\mathrm{H}_{\sigma}^{1}$.Using this bound recursively, we obtain

$\left\|\left(\nabla \mathrm{Y}^{\mathrm{i}+1}, \nabla \mathrm{Z}^{\mathrm{i}+1}\right)\right\|_{2}^{2} \leq\left(\mathrm{C} v^{-3} \mathrm{M}\|\mathrm{f}\|_{\mathrm{x}}\right)^{i+1} 2 v^{-2}\|\mathrm{f}\|_{\mathrm{x}}^{2}$

In the last step, we have used the uniform bound on

$$
\left\|\left(\nabla \mathrm{U}^{\mathrm{i}}, \nabla \mathrm{B}^{\mathrm{i}}\right)\right\|_{2} \leq v^{-2}\|\mathrm{f}\|_{\mathrm{x}}^{2}
$$

If $\|\mathrm{f}\|_{\mathrm{x}}<\frac{v^{3}}{\mathrm{CM}}$ where $\mathrm{C}$ is the same as above then $\left(\mathrm{Y}^{\mathrm{i}}, \mathrm{Z}^{\mathrm{i}}\right)$ tends to zero in $\dot{\mathrm{H}}_{\sigma}^{1} \times \dot{\mathrm{H}}_{\sigma}^{1}$. This implies that $\left(\widetilde{\mathrm{U}^{\mathrm{i}}, \mathrm{B}^{\mathrm{i}}}\right)$ is a Cauchy sequence. We denote its limit by $(\widetilde{\mathrm{U}, \mathrm{B}})$. This also ensures $(\widetilde{\mathrm{U}, \mathrm{B}}) \leq \mathrm{M}$. Using standard argument we can now show that $(\widetilde{\mathrm{U}, \mathrm{B}})$ is a solution of $(1.2)$.

To see that $(\widetilde{\mathrm{U}, \mathrm{B}})$ is the unique solution of (1.2) among all solutions which satisfy (2.1) \& have finite $\mathrm{L}^{2} \times \mathrm{L}^{2}$ norms , let $(\mathrm{U}, \mathrm{B})$ be any other solution which satisfies $(2.1) \&$ has a finite $\mathrm{L}_{\sigma}^{2} \times \mathrm{L}_{\sigma}^{2}$ norm.

The difference $(\mathrm{Y}, \mathrm{Z})=(\mathrm{U}, \mathrm{B})-(\widetilde{\mathrm{U}, \mathrm{B}})$ solves

$$
\begin{aligned}
& \mathrm{U} \cdot \nabla \mathrm{Y}+\mathrm{Y} \cdot \widetilde{\nabla U}-\mathrm{B} \cdot \nabla \mathrm{Z}-\mathrm{Z} \cdot \widetilde{\nabla \mathrm{B}}+\nabla \mathrm{p}=v \nabla \mathrm{Y} . \\
& \mathrm{U} \cdot \nabla \mathrm{Z}+\mathrm{Y} \cdot \widetilde{\nabla B}-\mathrm{B} \cdot \nabla \mathrm{Y}-\mathrm{Z} \cdot \widetilde{\nabla U} \quad=\lambda \nabla \mathrm{Z} .
\end{aligned}
$$

Multiplying the equation (3.27A) by $\mathrm{Y} \&(3.27 \mathrm{~B})$ by $\mathrm{Z}$ and then proceeding in the same manner as in the above proof ,we get

$$
\|(\nabla \mathrm{Y}, \nabla \mathrm{Z})\|_{2}^{2} \leq C v^{-3} \mathrm{M}\|\mathrm{f}\|_{\mathrm{X}}\|(\nabla \mathrm{Y}, \nabla \mathrm{Z})\|_{2}^{2}
$$

The assumption on $\mathrm{f}$ guarantees that if

$$
\|\mathrm{f}\|_{\mathrm{X}}<\frac{v^{3}}{\mathrm{CM}} \text { then }(\mathrm{Y}, \mathrm{Z}) \text { must vanish }
$$

this implies that the solution is unique.

\section{Conclusion:}

By using Fourier splitting technique developed by M. Schonbek, and a bootstrap argument, we have proved the existence of at least one solution with finite Dirichlet Integral to steady state Magnetohydrodynamic equations in the whole space. Further, we have shown that these solutions are 
unique among all solutions with finite energy and finite Dirichlet Integral. In future, building up upon these techniques, we intend to prove similar results for non-steady incompressible MHD flows. The question of global regularity of MHD flows still remains to be settled. We hope that our results proved here will throw some light on this problem, at least in the steady case.

\section{References:}

[1] G. Duvaut and J.L. Lions, Arch. Rat. Mech. Anal., 46 (1972), 241-279.

[2] E. Sanchez Palencia, Journal de Mechanique, 8(4) (1969), 509-541.

[3] M. Sermange and R.Temam, Commu. Pure Appl. Math., XXXVI (1983),635-664.

[4] C. Bjorland and M. Schonbek, Nonlinearity, Vol. 22, (2009) 1615-1637.

[5] Yong Zhou, Ann. Inst. HenriPoincaré - AN 24 (2007) 491-505

[6] Jiahong Wu, J. Math. Fluid Mech. 13 (2011), 295-305

[7] QunyiBie, Qiru Wang and Zhengan Yao, Regularity criteria for the 3D MHD equations in term of velocity, arXiv: 1312.1012 v1 [math.AP] 4 Dec. 2013

[8] P. Constantin and C. Foias.Navier-Stokes equations. Chicago Lectures in Mathematics. Universityof Chicago Press, Chicago, IL, 1988.

[9] R. Finn. On steady-state solutions of the Navier-Stokes partial differential equations. Arch.Rational Mech. Anal., 3:381-396 (1959), 1959.

[10] H. Fujita. On the existence and regularity of the steady-state solutions of the NavierStokestheorem. J. Fac. Sci. Univ. Tokyo Sect. I, 9:59-102 (1961)

[11] O. A. Ladyzhenskaya. The mathematical theory of viscous incompressible flow.Second Englishedition, revised and enlarged.Translated from the Russian by Richard A. Silverman andJohn Chu.Mathematics and its Applications, Vol. 2.Gordon and Breach Science Publishers,New York, 1969.

[12] J. Leray. 'etude de diverses 'equations int'egrales non lin'eaireset de quelquesprobl'emesquepsel'hydrodynamique. J. de Math. Pureset appl., 12:1-82, 1933.

[13] R. Temam. Navier-Stokes equations.AMS Chelsea Publishing, Providence, RI, 2001. Theoryand numerical analysis, Reprint of the 1984 edition.

[14] S D Mohagaonkar and R V Saraykar, L2 - Decay for the solutions of MHD Equations, Jour.Math.Phys.Sci Vol.23 no.1, p 35-55, 1989 